\title{
Odd-odd nuclei as the core-particle-hole systems and chirality
}

\author{
S.G. Rohoziński ${ }^{1, a}$, L. Próchniak ${ }^{2}$, K. Starosta ${ }^{3}$, and Ch. Droste ${ }^{4}$ \\ 1 Institute of Theoretical Physics, Faculty of Physics, University of Warsaw, Hoża 69, 00-681 Warsaw, Poland \\ 2 Institute of Physics, Maria Curie-Skłodowska University, Pl. M. Curie-Skłodowskiej 1, 20-031 Lublin, Poland \\ 3 Department of Chemistry, Simon Fraser University, 8888 University Drive, Burnaby, British Columbia, V5A1S6, Canada \\ 4 Institute of Experimental Physics, Faculty of Physics, University of Warsaw, Hoża 69, 00-681 Warsaw, Poland
}

Received: 29 January 2011 / Revised: 6 June 2011

Published online: 4 August 2011

(C) The Author(s) 2011. This article is published with open access at Springerlink.com

Communicated by A. Schwenk

\begin{abstract}
Odd-odd nuclei treated as core-particle-hole systems with various collective cores and various particle-hole configurations are investigated within the Core-Particle-Hole Coupling (CPHC) model. A new symmetry, called the $S$-symmetry, is identified as a combination of the $\alpha$-parity of the collective core and the proton-neutron symmetry of the valence proton and neutron in particle-hole configurations involving single-particle states with the same quantum numbers. It is found that the $S$-symmetric odd-odd nuclei show signatures which are usually considered as fingerprints of nuclear chirality, namely doublet band structure with a particular pattern of electromagnetic transitions. Reported results imply that the rigid rotor with a symmetric valence proton-neutron configuration is only a special case of the system with the novel $S$-symmetry. Therefore, it is an open question whether the chiral fingerprints discussed so far identify uniquely the orthogonal coupling of angular momentum in the intrinsic system.
\end{abstract}

\section{Introduction}

Frauendorf and Meng [1] were the first to suggest that the two handednesses or chiralities of three angular momentum pseudovectors appearing in a nuclear system can explain some rotational phenomena in its structure. The existence of the hypothetical left- and right-handed intrinsic nuclear states implies the existence of two nearly degenerate chiral partner bands with the same parity and similar electromagnetic properties, like the $M 1$ and $E 2$ spectroscopic moments, and the intra- and inter-band $M 1$ and $E 2$ transitions. Such states with the broken chiral symmetry can be found using the Tilted Axis Cranking (TAC) approach to the odd-odd nuclei [1-5].

The TAC model uses an intrinsic reference frame defined by the principal axes of the triaxially deformed nucleus of interest. The doublet bands in odd-odd nuclei can also be studied with respect to a laboratory reference frame using a Core-Quasiparticle-Pair Coupling model [6, 7]. When the pairing correlations may be neglected the model is reduced to a form with the particles (and holes) instead of the quasiparticles. One of such versions is the Core-Particle-Hole Coupling (CPHC) model [8] in which the odd-odd nucleus is treated as the system containing the even-even core, the odd proton and the odd neutronhole. Such a three-body system is a natural candidate for the one with the chiral geometry.

\footnotetext{
${ }^{a}$ e-mail: Stanislaw-G.Rohozinski@fuw.edu.pl
}

In fact, the chiral geometry was demonstrated for the first time in the case of the system containing the rigid triaxial rotor, and the proton and the neutron-hole, both on the same high- $j$ shell in the deformed mean field [1]. Within the quasi-classical picture the proton and the neutron-hole circles around the short and the long principal axis of the rotor, respectively, whereas the rotor itself rotates around its intermediate axis. Then, the angular momenta of the three bodies are directed along the principal axes and form either the left-handed or the righthanded system depending on the directions of circulation.

Subsequently, various versions of the rotor- quasiparticle/ particle-hole model were applied to different nuclei, and the chirality phenomenon was investigated [814]. The rigidity and the maximal triaxiality $\left(\gamma=30^{\circ}\right)$ of the core and the symmetric particle-hole configuration of the odd particles leads to the staggering in values of the reduced transition probabilities of the $\Delta I=1 M 1$ and $E 2$ intra-band and inter-band transitions within the chiral partner bands. From the beginning the $B(M 1)$ staggering was considered as an aspect of chirality. However, it turned out that this feature of the electromagnetic transitions does not result from the chirality itself but it follows rather from possible additional symmetries possessed by chiral systems $[15,16]$. This was recently stated clearly in [17]. Anyway, even quite recently one can find opinions that "the $B(M 1)$ staggering is associated strongly with the characters of nuclear chirality" $[18,19]$. Also, the chiral systems manifest some other characteristic features 
coming from a particular structure of the system rather than from chirality itself.

Up to now it was found that the signatures of chirality, especially the electromagnetic properties, are manifested most distinctly in the two nuclei in the region $A \approx 130$, namely ${ }^{126} \mathrm{Cs}$ and ${ }^{128} \mathrm{Cs}[20,21]$.

A question arises on which properties of the nucleus are sine qua non for the appearance of signatures suggested as chiral fingerprints. The current work addresses this question. In our previous paper [22] we demonstrated that the odd-odd nucleus with the $\gamma$-unstable core and the $\pi h_{11 / 2} \otimes \nu h_{11 / 2}^{-1}$ particle-hole configuration manifests all the main signatures of chirality including the phenomenon of staggering in electromagnetic transition rates. In the present study we consider fictitious odd-odd nuclei with various cores described by the Bohr collective Hamiltonian, and different particle-hole configurations to look for the characteristics in their spectra which resemble main properties of the chiral systems. We try to formulate as comprehensive as possible sufficient conditions for the appearance of these properties. As shown below, it is observed that the main chiral fingerprints including the staggering appear in a significant class of Hamiltonians which possess the novel $S$-symmetry identified in this paper. This result may imply that the chiral fingerprints discussed so far may not uniquely identify the orthogonal coupling of the angular momentum in the intrinsic system. We do not go into the question of whether the systems considered here possess the chiral geometry. We ascertain only that the main characteristics of chirality can be explained by the $S$-symmetry as well. The possible impact of the $S$-symmetry on the geometry of angular momentum coupling and further investigation of observables unique to nuclear chirality is planned as a follow-up of the studies presented here.

In sect. 2, the CPHC model and the grounds of calculations are briefly presented. We mention the description of the core through the collective model (sect. 2.1) and define the $\alpha$-symmetry of the Bohr Hamiltonian (sect. 2.2). The proton-neutron symmetry of the particle-hole configuration (sect. 2.3) and the combined symmetry of the entire odd-odd system (sect. 2.4) are presented. The results of calculations are discussed in sect. 3 . Characteristics of the $\alpha$-symmetric cores used in the calculations are presented in sect. 3.1. In sect. 3.2 the properties of the odd-odd nuclei with these $\alpha$-symmetric cores, and the proton and neutron-hole either on the same orbital (sect. 3.2.1) or on the different orbitals (sect. 3.2.2) are reported. The $\alpha$ asymmetric cores are discussed in sect. 3.3. The results for the odd-odd nuclei with $\alpha$-asymmetric cores and the symmetric particle-hole configuration are shown in sect. 3.4. In sect. 4 we draw conclusions from the present research.

\section{Odd-odd nuclei as core-particle-hole systems}

In the present paper we treat an odd-odd nucleus as a coreproton-neutron three-body system interacting through the separable quadrupole-quadrupole forces. In general, such an approach containing also the pairing interaction is called the Core-Quasiparticle-Pair Coupling model as mentioned in the Introduction. Here we use a simplified version of the model called the Core-Particle-Hole Coupling (CPHC) model. All details of the CPHC model, which we apply to modelling odd-odd nuclei, are given in refs. $[8,22]$. We assume that the pairing correlations can be neglected, and the odd valence particles are a proton and a neutron-hole. The proton can fill a state from a set of the unoccupied single-particle orbitals $\pi \rho=\pi n_{\rho} l_{j_{\rho}}$ with energies $\varepsilon_{\pi}(\rho)$. The neutron-hole can vacate a state from a set of the occupied single-particle states $\nu \sigma^{-1}=\nu n_{\sigma} l_{j_{\sigma}}^{-1}$ with energies $\varepsilon_{\nu}(\sigma)$. Because of a difference between the numbers of protons and neutrons the sets of the proton unoccupied states and the neutron occupied states can coincide with each other either partially or completely.

Within the CPHC model the $i$-th state with spin $I$ of the odd-odd nucleus with $Z$ protons and $N$ neutrons can be expanded in the form:

$$
\begin{aligned}
& \left|Z, N ; I_{i} M_{I}\right\rangle= \\
& \quad \sum_{\rho, \sigma} \sum_{L, R, r} U_{I i}(\rho, \sigma, L, R, r) \\
& \quad \times\left[\left[a_{\pi \rho}^{\dagger} \times \tilde{a}_{\nu \sigma}\right]_{L} \times\left|Z-1, N+1 ; R_{r}\right\rangle\right]_{I M_{I}},
\end{aligned}
$$

where $a_{\pi \rho}^{\dagger}$ and $\tilde{a}_{\nu \sigma}$ are the creation operators of the proton and the neutron-hole, respectively, $\left|Z-1, N+1 ; R_{r}\right\rangle$ is the $r$-th basis state with spin $R$ and energy $E\left(R_{r}\right)$ of the even-even core, and $U_{I i}(\rho, \sigma, L, R, r)$ are the expansion coefficients. The eigenvalue equation for coefficients $U_{I i}(\rho, \sigma, L, R, r)$ is obtained by using the techniques of the CPHC model and is given in $[8,22]$. The corresponding Hamiltonian matrix for a given $I$ takes the form $[8,22]$

$$
\begin{aligned}
& \mathcal{H}_{I}\left(\rho, \sigma, L, R, r \mid \rho^{\prime}, \sigma^{\prime}, L^{\prime}, R^{\prime}, r^{\prime}\right)= \\
& \left(E\left(R_{r}\right)+\varepsilon_{\pi}(\rho)-\varepsilon_{\nu}(\sigma)\right) \delta_{\rho \rho^{\prime}} \delta_{\sigma \sigma^{\prime}} \delta_{L L^{\prime}} \delta_{R R^{\prime}} \delta_{r r^{\prime}} \\
& -\chi(-1)^{R+I} \sqrt{2 L+1} \sqrt{2 L^{\prime}+1}\left\{\begin{array}{ccc}
L^{\prime} & 2 & L \\
R & I & R^{\prime}
\end{array}\right\}\left\langle R_{r}\|Q\| R_{r^{\prime}}^{\prime}\right\rangle \\
& \times\left[(-1)^{j_{\rho}+j_{\sigma}}\left\{\begin{array}{ccc}
j_{\rho^{\prime}} & 2 & j_{\rho} \\
L & j_{\sigma} & L^{\prime}
\end{array}\right\}\left\langle\rho\left\|q_{\pi}\right\| \rho^{\prime}\right\rangle \delta_{\sigma \sigma^{\prime}}\right. \\
& \left.-(-1)^{j_{\rho}+j_{\sigma^{\prime}}+L+L^{\prime}}\left\{\begin{array}{ccc}
j_{\sigma^{\prime}} & 2 & j_{\sigma} \\
L & j_{\rho} & L^{\prime}
\end{array}\right\} \delta_{\rho \rho^{\prime}}\left\langle\sigma\left\|q_{\nu}\right\| \sigma^{\prime}\right\rangle\right] \\
& +\chi(-1)^{j_{\rho^{\prime}}+j_{\sigma}+L}\left\{\begin{array}{ccc}
j_{\sigma^{\prime}} & 2 & j_{\sigma} \\
j_{\rho} & L & j_{\rho^{\prime}}
\end{array}\right\} \\
& \times \delta_{L L^{\prime}} \delta_{R R^{\prime}} \delta_{r r^{\prime}}\left\langle\rho\left\|q_{\pi}\right\| \rho^{\prime}\right\rangle\left\langle\sigma\left\|q_{\nu}\right\| \sigma^{\prime}\right\rangle
\end{aligned}
$$

where $\chi$ is the quadrupole coupling constant, and $Q, q_{\pi}$ and $q_{\nu}$ are the mass quadrupole operator of the core, and the single-particle quadrupole operator $\left(r^{2} Y_{2}\right)$ of the proton and the neutron, respectively. In the present calculations we assume that the proton occupies the $\pi 1 h_{11 / 2}$ orbital and the neutron-hole can occupy the one out of three orbitals: $\nu 1 h_{11 / 2}^{-1}, \nu 1 g_{9 / 2}^{-1}, \nu 1 f_{7 / 2}^{-1}$. In the following we shall omit the numbers of protons and neutrons in the notation of the even-even core and the odd-odd nucleus states, and 
label the single-particle orbits $n l_{j}$ only by their total angular momenta $j$. The state (1) obtained in the calculations is then denoted in the following form:

$$
\begin{aligned}
& \left|I_{i} M_{I}\right\rangle= \\
& \sum_{L, R, r} U_{I i}(\rho, \sigma, L, R, r)\left|\left(\pi j_{\rho} \nu j_{\sigma}^{-1}\right) L R_{r} ; I_{i} M_{I}\right\rangle .
\end{aligned}
$$

A similar description of the odd-odd nuclei treated as the three-body systems interacting with the quadrupolequadrupole forces is used in [23].

\subsection{Description of the even-even core}

We assume that the core states $\left|R_{r} M_{R}\right\rangle$ are eigenstates of the Bohr Hamiltonian (cf., e.g., [24]) in the following form:

$$
\begin{aligned}
& H(\beta, \gamma, \Omega)= \\
& -\frac{1}{2 B_{\beta \beta}} \frac{1}{\beta^{4}} \frac{\partial}{\partial \beta}\left(\beta^{4} \frac{\partial}{\partial \beta}\right)-\frac{1}{2 B(\gamma)} \frac{\Lambda^{2}(\gamma, \Omega)}{\beta^{2}}+V(\beta, \gamma),
\end{aligned}
$$

with a constant inertial parameter $B_{\beta \beta}$. The $S O(5)$ Casimir operator in (4) is

$$
\begin{aligned}
& \Lambda^{2}(\gamma, \Omega)= \\
& \frac{1}{\sin 3 \gamma} \frac{\partial}{\partial \gamma}\left(\sin 3 \gamma \frac{\partial}{\partial \gamma}\right)-\sum_{k=1}^{3} \frac{R_{k}^{2}(\Omega)}{\sin ^{2}(\gamma-2 \pi k / 3)} .
\end{aligned}
$$

Variables $\beta$ and $\gamma$ are the Bohr deformation parameters, $\Omega$ stands for the three Euler angles of orientation of the body-fixed system and $R_{k}(\Omega)$ for $k=1,2,3$ are the three (dimensionless) intrinsic components of angular momentum. To investigate various phenomena of the $\gamma$-susceptibility of the core we parametrize the potential $V(\beta, \gamma)$ and the inertial function $B(\gamma)$ in the following way:

$$
\begin{aligned}
V(\beta, \gamma)= & \frac{1}{2} V_{C} \beta^{2}+\left(G+h_{1} \cos 3 \gamma+h_{2}\left(\cos ^{2} 3 \gamma-1\right)^{\kappa}\right) \\
& \times\left(\exp \left(-\beta^{2} / d^{2}\right)-1\right)
\end{aligned}
$$

with a number of parameters $V_{C}, G, h_{1}, h_{2}, \kappa$ and $d$ relevant for a specific behaviour of the potential, and

$$
B(\gamma)=b_{0}+b_{1} \cos 3 \gamma
$$

with constant $b_{0}$ and $b_{1}$. The values of the parameters used in our previous calculations [22] were: $Z-1=56$ (the core atomic number is supposed to be $Z-1$, cf. eq. (1)), the mass number $A=128, b_{0}=105 / \mathrm{MeV}, B_{\beta \beta}=250 / \mathrm{MeV}$, $V_{C}=109.49 \mathrm{MeV}, G=8.605 \mathrm{MeV}, d=0.2511, h_{1}=$ $h_{2}=b_{1}=0$. In the present calculations we vary the values of parameters $h_{1}, h_{2}, b_{1}$ to have different potentials and kinetic energies with a different characteristic $\gamma$-dependence. To keep always the same scale we fit other parameters to every set $\left(h_{1}, h_{2}, b_{1}\right)$ so as to obtain the equilibrium value of $\beta$ about 0.25 , and the values of energy of the lowest excited state $E\left(2_{1}^{+}\right) \approx 0.35 \mathrm{MeV}$ and reduced transition probability $B\left(E 2 ; 2_{1}^{+} \rightarrow 0_{1}^{+}\right) \approx 0.28 e^{2} b^{2}$ close to the experimental values of $E\left(2_{1}^{+}\right)$and $B\left(E 2 ; 2_{1}^{+} \rightarrow 0_{1}^{+}\right)$ for ${ }_{56}^{128} \mathrm{Ba}$.

The rigid rotor Davydov-Filippov (DF) Hamiltonian [25]

$$
H_{\mathrm{DF}}(\Omega ; \beta, \gamma)=\frac{1}{8 B \beta^{2}} \sum_{k=1}^{3} \frac{R_{k}^{2}(\Omega)}{\sin ^{2}(\gamma-2 \pi k / 3)},
$$

with constant $B$, where the only dynamical variables are the three Euler angles, $\Omega$, can be treated as a limiting case of $H$ of (4) for the infinite stiffness against $\beta$ and $\gamma$. Then, $\beta$ and $\gamma$, in spite of the same notation, become constant parameters and not dynamical variables. Together with $B$ they fix the three moments of inertia.

\subsection{The $\alpha$-parity of the core}

The Wilets-Jean Hamiltonian (WJ) [26], which we used in [22] for the description of the $\gamma$-soft core, is invariant under the $S O(5)$ group of rotations in the five-dimensional space of the laboratory quadrupole coordinates

$$
\begin{aligned}
\alpha_{\mu}(\beta, \gamma, \Omega)= & {\left[D_{\mu 0}^{2}(\Omega) \beta \cos \gamma\right.} \\
& \left.+\frac{1}{\sqrt{2}}\left(D_{\mu 2}^{2}(\Omega)+D_{\mu-2}^{2}(\Omega)\right) \beta \sin \gamma\right]
\end{aligned}
$$

where $D_{\mu \mu^{\prime}}^{\lambda}(\Omega)$ are the Wigner functions [27]. Obviously, it remains invariant under the $O(5)$ group of orthogonal transformations in the five-dimensional space, which arises from $S O(5)$ by addition of the five-dimensional inversion $P_{\alpha}$ changing the signs of $\alpha_{\mu}$ for $\mu=-2, \ldots,+2$. The inversion $P_{\alpha}$ transforms the intrinsic collective variables

$$
(\beta, \gamma, \Omega) \rightarrow(\beta, \gamma \pm \pi, \Omega)
$$

or, when $\gamma$ is restricted to one sextant $0 \leq \gamma \leq \pi / 3$,

$$
(\beta, \gamma, \Omega) \rightarrow\left(\beta, \pi / 3-\gamma, R_{x}(\pi / 2) \Omega\right),
$$

where $R_{x}(\pi / 2)$ is the rotation by $\pi / 2$ around the bodyfixed axis $x$. There exists a large class of the Bohr Hamiltonians invariant under $P_{\alpha}$ alone. General conditions for the Bohr Hamiltonian to be even under inversion $P_{\alpha}$ are mentioned in [28]. Hamiltonian (4) is the $P_{\alpha}$ invariant provided $b_{1}=h_{1}=0$. In the DF model in which the dynamical variables are restricted to the Euler angles $\Omega$ the transformation $R_{x}(\pi / 2)$ plays the role of $P_{\alpha}$. It is seen from (11) that for the DF Hamiltonian we have

$$
\begin{aligned}
& R_{x}(\pi / 2) H_{\mathrm{DF}}(\Omega ; \beta, \gamma) R_{x}^{\dagger}(\pi / 2)= \\
& H_{\mathrm{DF}}(\Omega ; \beta, \pi / 3-\gamma) .
\end{aligned}
$$

Hence, the DF Hamiltonian is $R_{x}(\pi / 2)$ invariant only for $\gamma=\pi / 6$. In the case of the $P_{\alpha}$ invariant Bohr Hamiltonians (or the $R_{x}(\pi / 2)$ invariant DF's Hamiltonian) the core 
states $\left|R_{r} M_{R}\right\rangle=\left|R_{r} p\left(R_{r}\right) M_{R}\right\rangle$ have a definite eigenvalue $p= \pm 1$ of $P_{\alpha}$ which we will call the $\alpha$-parity quantum number (the $R^{5}$ parity according to [29]). Bés [30] was the first to use the $p$ quantum number called a bit misleadingly the $\gamma$-parity. For the eigenstates of the WJ Hamiltonian which have a definite boson's seniority $\lambda$ [31] the $\alpha$-parity is $p(\lambda)=(-1)^{\lambda}$.

The reduced matrix elements of $\cos 3 \gamma$ within the states of the same $\alpha$-parity $p$ vanish:

$$
\left\langle R_{r^{\prime}} p\|\cos 3 \gamma\| R_{r} p\right\rangle=0 .
$$

The collective $E 2$ operator

$$
\begin{aligned}
& M(E 2 ; \mu)=\frac{3}{4 \pi} Z e R_{0}^{2} \alpha_{\mu}(\beta, \gamma, \Omega)= \\
& \frac{3}{4 \pi} Z e R_{0}^{2}\left[D_{\mu 0}^{2}(\Omega) \beta \cos \gamma\right. \\
& \left.+\frac{1}{\sqrt{2}}\left(D_{\mu 2}^{2}(\Omega)+D_{\mu-2}^{2}(\Omega)\right) \beta \sin \gamma\right]
\end{aligned}
$$

has the negative $\alpha$-parity, and thus its reduced matrix elements have similar properties,

$$
\left\langle R_{r^{\prime}}^{\prime} p\|M(E 2)\| R_{r} p\right\rangle=0
$$

On the other hand the collective $M 1$ operator,

$$
\begin{aligned}
& M(M 1 ; \mu)= \\
& \sqrt{\frac{3}{4 \pi}} \mu_{N} g_{R}\left[-\frac{1}{\sqrt{2}}\left(D_{\mu 1}^{1}(\Omega)-D_{\mu-1}^{1}(\Omega)\right) R_{1}(\Omega)\right. \\
& +\frac{i}{\sqrt{2}}\left(D_{\mu 1}^{1}(\Omega)+D_{\mu-1}^{1}(\Omega)\right) R_{2}(\Omega) \\
& \left.+D_{\mu 0}^{1}(\Omega) R_{3}(\Omega)\right]
\end{aligned}
$$

has positive $\alpha$-parity and is proportional to the collective angular momentum, and therefore only its diagonal matrix elements can have nonvanishing values. It means that

$$
\begin{aligned}
& \left\langle R_{r^{\prime}}-p\|M(M 1)\| R_{r} p\right\rangle=0, \\
& \left\langle(R \pm 1)_{r^{\prime}} p^{\prime}\|M(M 1)\| R_{r} p\right\rangle=0,
\end{aligned}
$$

for any $r, r^{\prime}$ and $p, p^{\prime}$.

\subsection{The proton-neutron symmetry}

Let us define a transformation $C_{\pi \nu}$ which acts on the states of the valence nucleons and interchanges the proton and the neutron-hole $\pi \rightleftharpoons \nu^{-1}$ (cf. [15]), and to be more precise, it exchanges the proton in the state $\rho$ for the neutron-hole in the same state $\rho: \pi \rho \rightarrow \nu \rho^{-1}$ and vice versa. The proton-neutron exchange operator $C_{\pi \nu}$ acts on the basis states $\left|\left(\pi j_{\rho} \nu j_{\sigma}^{-1}\right) L R_{r} ; I M_{I}\right\rangle$ of (3) as follows:

$$
\begin{aligned}
& C_{\pi \nu}\left|\left(\pi j_{\rho} \nu j_{\sigma}^{-1}\right) L R_{r} ; I M_{I}\right\rangle= \\
& (-1)^{j_{\rho}+j_{\sigma}-L}\left|\left(\pi j_{\sigma} \nu j_{\rho}^{-1}\right) L R_{r} ; I M_{I}\right\rangle,
\end{aligned}
$$

which shows that the operation $C_{\pi \nu}$ means the interchange of the proton and the neutron states. It follows from (19) that state $\left|\left(\pi j_{\rho} \nu j_{\rho}^{-1}\right) L R_{r} ; I M_{I}\right\rangle$ is an eigenstate of $C_{\pi \nu}$ belonging to eigenvalue $(-1)^{L+1}$. The matrix elements of $C_{\pi \nu}$ within the basis states of a definite $I$ read

$$
\begin{aligned}
& \mathcal{C}_{\pi \nu}\left(\rho^{\prime}, \sigma^{\prime}, L^{\prime}, R^{\prime}, r^{\prime} \mid \rho, \sigma, L, R, r\right)= \\
& (-1)^{j_{\rho}+j_{\sigma}-L} \delta_{\rho^{\prime} \sigma} \delta_{\sigma^{\prime} \rho} \delta_{L^{\prime} L} \delta_{R^{\prime} R} \delta_{r^{\prime} r}
\end{aligned}
$$

\subsection{Combined symmetry of the core-particle-hole system}

Obviously, $C_{\pi \nu}$ commutes with the $\alpha$-parity $P_{\alpha}$ and $C_{\pi \nu}^{2}=$ $1, P_{\alpha}^{2}=1$. Since the mass quadrupole operator of the core,

$$
Q_{\mu}(\alpha)=\frac{3}{4 \pi} A R_{0}^{2} \alpha_{\mu}
$$

has the negative $\alpha$-parity the operator $P_{\alpha}$ acting on the Hamiltonian matrix (2) exchanges the signs of the core matrix elements $\left\langle R_{r}\|Q\| R_{r^{\prime}}^{\prime}\right\rangle$ for the opposite ones. It can be checked using (2) and (20) that

$$
\mathcal{C}_{\pi \nu} P_{\alpha} \mathcal{H}_{I} P_{\alpha}^{\dagger} \mathcal{C}_{\pi \nu}^{\dagger}=\mathcal{H}_{I}
$$

provided the core alone is the $\alpha$-symmetric, and $\varepsilon_{\pi}(\rho)-$ $\varepsilon_{\pi}(\sigma)=\varepsilon_{\nu}(\rho)-\varepsilon_{\nu}(\sigma)$, i.e. the single-particle energy levels of the proton and the neutron either are the same or shifted as a whole with respect to one another. It means that operator $S=P_{\alpha} C_{\pi \nu}$ is a combined symmetry of the quadrupole interaction and thus the symmetry of the oddodd nucleus treated as an interacting core-particle-hole system. Then, the states with a given spin $I$ of the oddodd nucleus $\left|I_{i} M_{I}\right\rangle=\left|I_{i} s\left(I_{i}\right) M_{I}\right\rangle$ have a definite quantum number $s= \pm 1$, the eigenvalue of $S$. Operation $S$ is a generalisation of operation $A$ of refs. $[15,16]$.

The electric quadrupole operator for the odd-odd nucleus is equal to

$$
\mathcal{M}(E 2 ; \mu)=M(E 2 ; \mu)+e_{\pi} q_{\pi \mu}-e_{\nu} q_{\nu \mu},
$$

where $M(E 2)$ is the $E 2$ moment of the core (14), and $e_{\pi}$ and $e_{\nu}$ are the electric effective charges of the proton and the neutron, respectively. The minus sign in front of the quadrupole moment of the neutron comes from the fact that the quadrupole moment of the hole has the sign opposite to that of the particle (cf. [27]). The core part is an odd operator with respect to $P_{\alpha}$ and $S$. Should the effective charges of the proton and neutron be equal to each other, the entire $\mathcal{M}(E 2)$ operator would be odd with respect to $S$ too, since the single-particle part is odd with respect to $C_{\pi \nu}$. In reality it is not the case. In the present calculations we put $e_{\pi}=e, e_{\nu}=0$. Fortunately, the collective $E 2$ operator of the core has usually much bigger matrix elements than the single-particle ones. Therefore, the reduced $E 2$ transition probabilities are much bigger for transitions between the states with opposite $s$ than those for transitions between the same $s$ states. 
The magnetic dipole operator for the odd-odd nucleus has the form:

$$
\begin{aligned}
& \mathcal{M}(M 1 ; \mu)=M(M 1 ; \mu) \\
& +\sqrt{\frac{3}{4 \pi}} \mu_{N}\left[g_{\pi l} l_{\pi \mu}+g_{\pi s} s_{\pi \mu}+g_{\nu l} l_{\nu \mu}+g_{\nu s} s_{\nu \mu}\right],
\end{aligned}
$$

where $M(M 1)$ is given by (16), $l_{\pi \mu}, s_{\pi \mu}$ and $l_{\nu \mu}, s_{\nu \mu}$ are the spherical components of the proton and neutron orbital angular momentum and spin vectors, respectively, and $g_{\pi l}, g_{\pi s}$ and $g_{\nu l}, g_{\nu s}$ are the effective orbital and spin $g$-factors of the proton and neutron, respectively. The vector of total angular momentum of the odd-odd nucleus is

$$
I_{\mu}=R_{\mu}+l_{\pi \mu}+s_{\pi \mu}+l_{\nu \mu}+s_{\nu \mu} .
$$

Using (25) to eliminate the angular momentum of the core from (24) we have (cf. [32]):

$$
\begin{aligned}
\mathcal{M}(M 1 ; \mu)= & \sqrt{\frac{3}{4 \pi}} \mu_{N}\left\{g_{R} I_{\mu}\right. \\
& +\left(g_{\pi l}-g_{R}\right) l_{\pi \mu}+\left(g_{\nu l}-g_{R}\right) s_{\pi \mu} \\
& \left.+\left(g_{\nu l}-g_{R}\right) l_{\nu \mu}+\left(g_{\nu s}-g_{R}\right) s_{\nu \mu}\right\} .
\end{aligned}
$$

The first term on the right-hand side of formula (26) gives rise to the magnetic dipole moments and has no contribution to the $M 1$ transitions because only the diagonal reduced matrix elements of it do not vanish. The remaining terms of (26) form a linear combination of the proton and neutron orbital angular momenta and spins with the coefficients being the differences of the corresponding particle $g$-factor and $g_{R}$. In the present calculations we use $g_{R}=(Z-1) / A=0.44, g_{\pi l}=1, g_{\pi s}=0.6 g_{\pi s}^{\text {free }}=3.35$, $g_{\nu l}=0$ and $g_{\nu s}=0.6 g_{\nu s}^{\text {free }}=-2.29$. Hence, we have

$$
\begin{array}{ll}
g_{\pi l}-g_{R}=0.56, & g_{\nu l}-g_{R}=-0.44, \\
g_{\pi s}-g_{R}=2.91, & g_{\nu s}-g_{R}=-2.73 .
\end{array}
$$

We see that the corresponding $g$-factor differences for the proton and the neutron differ in signs and have the absolute values fairly close to each other. In consequence, the $M 1$ transition operator is approximately odd with respect to $C_{\pi \nu}$, and thus to $S$, which means that the $M 1$ reduced matrix elements within the states of the opposite $s$ should be much bigger than those within the states of the same $s$ (see the figure in [28]).

\section{The calculations}

Our previous calculations performed in the frame of the CPHC model in [22] showed that the odd-odd nuclei need not have the rigid triaxial core to exhibit the features of chirality. The odd-odd nuclei with the $\gamma$-unstable cores also reveal the signs of chirality including also the $B(M 1)$ staggering. A few questions arise: what is a common feature of these two extreme cases of the $\gamma$-softness which manifests itself as a sign of chirality? Are there other cores which also have such a feature? Which orbitals should the
Table 1. Parameters $h_{2}$ and $V_{C}$ of the $\alpha$-symmetric potential used in the calculation of the core characteristics. Energy $E\left(2_{1}^{+}\right)$of the first-excited state and the reduced transition probability $B\left(E 2 ; 2_{1}^{+} \rightarrow 0_{1}^{+}\right)$calculated with these parameters are also shown.

\begin{tabular}{rrrc}
\hline$h_{2}$ & $\begin{array}{r}V_{C} \\
(\mathrm{MeV})\end{array}$ & $\begin{array}{r}E\left(2_{1}^{+}\right) \\
(\mathrm{MeV})\end{array}$ & $\begin{array}{c}B\left(E 2 ; 2_{1}^{+} \rightarrow 0_{1}^{+}\right) \\
\left(e^{2} b^{2}\right)\end{array}$ \\
\hline 4 & 143 & 0.355 & 0.273 \\
20 & 356 & 0.354 & 0.252 \\
\hline-4 & 103 & 0.354 & 0.269 \\
-8 & 110 & 0.356 & 0.245 \\
-20 & 128 & 0.333 & 0.206 \\
\hline
\end{tabular}

odd proton and neutron occupy? Below we present results of the CPHC calculations performed in the cases of different cores and different single-particle orbitals which address these questions. We will examine the roles of the $\alpha$-parity of the core, and the occupation of different orbits by the proton and the neutron-hole. To observe the chirality phenomenon in odd-odd nuclei we take, like in [22], a fictitious nucleus of $Z=57$ and $N=71$ with different cores and different particle-hole configurations. Most calculations are performed for the case of the proton-neutron symmetry when the odd proton and the neutron-hole occupy the $\pi 1 h_{11 / 2}$ and $\nu 1 h_{11 / 2}^{-1}$ orbits, respectively.

\subsection{The $\alpha$-symmetric cores}

To examine the role of rigidity of the core we perform the calculation with the $\alpha$-symmetric cores described by the Bohr Hamiltonian (4) with $b_{0}=105 / \mathrm{MeV}, b_{1}=0$ and $B_{\beta \beta}=250 / \mathrm{MeV}$, and the potential (6). The parameters of the potential used in the calculation are: $G=8.605 \mathrm{MeV}$, $h_{1}=0, \kappa=4, d=0.251$. The two remaining parameters of the potential, $h_{2}$ and $V_{C}$, have been changed in order to investigate the potentials with either different depths of the well in $\gamma\left(h_{2}>0\right)$ or different heights of the barrier in $\gamma\left(h_{2}<0\right)$ and, at the same time, to keep the values of $E\left(2_{1}^{+}\right)$and $B\left(E 2 ; 2_{1}^{+} \rightarrow 0_{1}^{+}\right)$close to the experimental values for ${ }_{56}^{128} \mathrm{Ba}$. We present results of the calculations of properties of the core for the selected values of $h_{2}$ and $V_{C}$ given in table 1 . Contour maps of the three potentials with parameter $h_{2}=20,0,-8 \mathrm{MeV}$ are shown in [33] (fig. 1). The dependence of the energy spectrum $E\left(R_{r}^{+}\right)$on the well depth parameter $h_{2}>0$ is shown in fig. 1 . The energy spectra given by the WJ and the DF models are shown for comparison. We see that the well of potential causes splitting of the WJ seniority multiplets. The rotational levels tend to the levels of the DF model. The vibrational ones (e.g., the $0_{2}^{+}$state) do not appear in the DF model. The reduced transition probabilities $B\left(E 2 ; R_{1}^{+} \rightarrow(R-\right.$ $2)_{1}^{+}$) within the yrast band calculated in cases a) - d) of fig. 1 are shown in fig. 2. When the depth of the well becomes bigger their values tend slowly away from the values given by the WJ model towards those given by the DF model. 


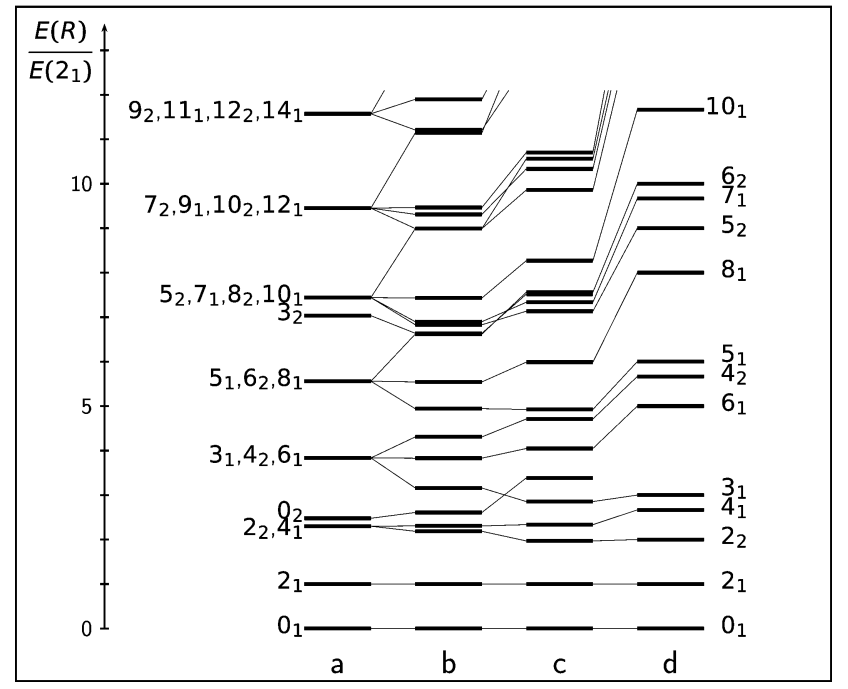

Fig. 1. Energy levels $E\left(R_{r}^{+}\right) / E\left(2_{1}^{+}\right)$with $r=1,2$, in units of the first-excited state calculated by using: a) the WJ model $\left(h_{2}=0\right)$, b) the potential with a well in variable $\gamma$ for $h_{2}=$ $4 \mathrm{MeV}, \mathrm{c}$ ) the potential with $\left.h_{2}=20 \mathrm{MeV}, \mathrm{d}\right)$ the DF model with $\gamma=\pi / 6$.

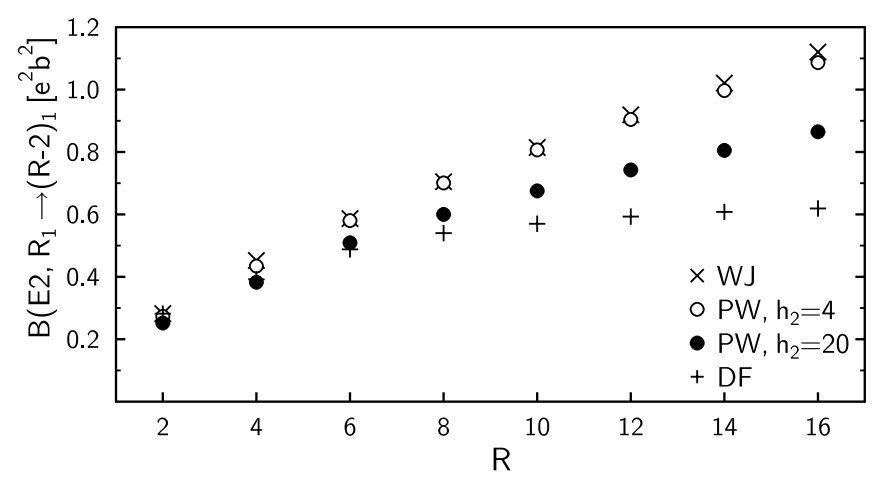

Fig. 2. Reduced transition probabilities $B\left(E 2 ; R_{1}^{+} \rightarrow(R-\right.$ $2)_{1}^{+}$) calculated in the cases a)-d) of fig. 1 .

At first glance one could expect that if there appears a barrier in the variable $\gamma$ of the collective potential, the properties of the core should be different from those of the core with the potential well. Let us see how it is in reality. Figure 3 shows, for a given $R$, the two lowest energy levels $E\left(R_{r}^{+}\right)(r=1,2)$ for the three heights of the barrier compared with those of the WJ model. Appearance of the barrier causes splitting of the WJ seniority multiplets. However, for high barriers the neighbouring levels of the same spin become degenerate. The reduced transition probabilities $B\left(E 2 ; R_{r}^{+} \rightarrow(R-2)_{r}^{+}\right)$with $r=1,2$ do not differ much from $B\left(E 2 ; R_{1}^{+} \rightarrow(R-2)_{1}^{+}\right)$given by the WJ model. It is seen in fig. 4 for the barrier height parameter $h_{2}=-8 \mathrm{MeV}$.

\subsection{Odd-odd nuclei with the $\alpha$-symmetric cores}

The properties of the odd-odd nucleus depend not only on the properties of the core but also on a configuration of the

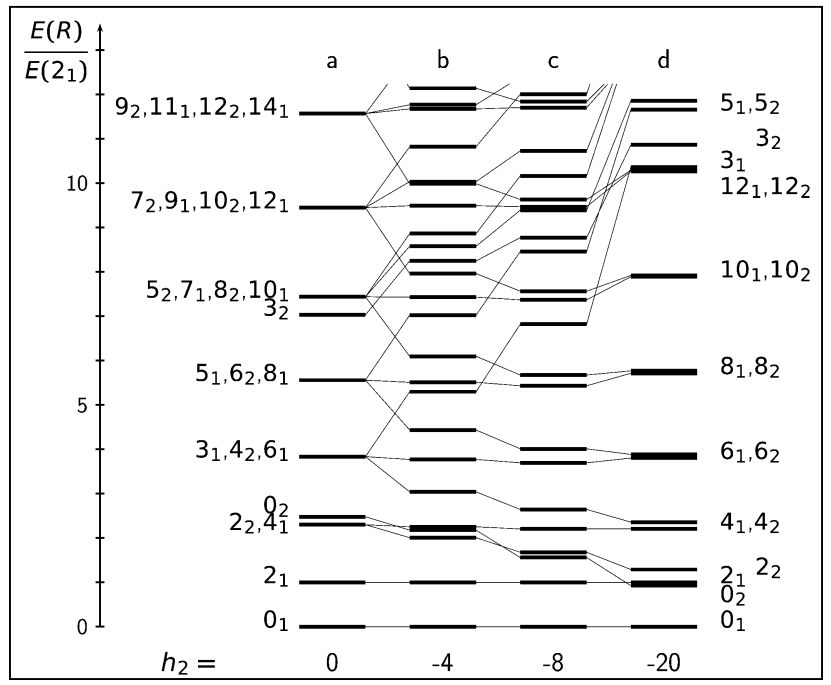

Fig. 3. Energy levels $E\left(R_{r}^{+}\right) / E\left(2_{1}^{+}\right)$with $r=1,2$, in units of the first-excited state calculated by using: a) the WJ model $\left(h_{2}=0\right)$, b) the potential with a barrier in variable $\gamma$ for $\left.h_{2}=-4 \mathrm{MeV}, \mathrm{c}\right)$ the potential with $h_{2}=-8 \mathrm{MeV}, \mathrm{d}$ ) the potential with $h_{2}=-20 \mathrm{MeV}$.

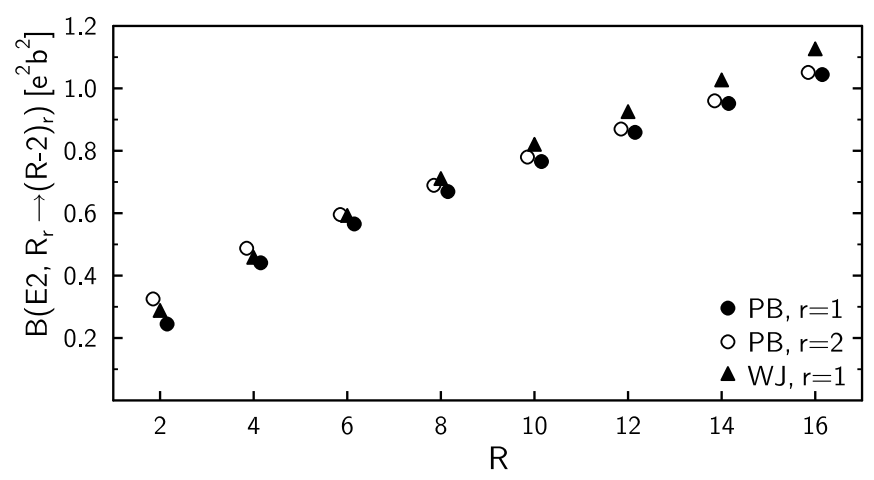

Fig. 4. Reduced transition probabilities $B\left(E 2 ; R_{r}^{+} \rightarrow(R-\right.$ $2)_{r}^{+}$) calculated in the case of $h_{2}=-8 \mathrm{MeV}$ for $r=1,2$ compared with $B\left(E 2 ; R_{1}^{+} \rightarrow(R-2)_{1}^{+}\right)$given by the WJ model.

odd particle and hole. Below we present, as an example, results of the CPHC calculations of the properties of oddodd nuclei with the different $\alpha$-symmetric cores and the different configurations of the proton and the neutronhole.

\subsubsection{Proton and neutron on the same orbital}

Let us take the odd-odd nucleus with an $\alpha$-symmetric core, and the odd proton and the odd neutron-hole which both occupy the $1 h_{11 / 2}$ single-particle orbit. Such a nucleus is said to be $S$-symmetric. The results of calculations for the two cores are to be presented. The collective potential energy of the one core (PW) has the well in $\gamma$ with $h_{2}=$ $20 \mathrm{MeV}$ and that of the other one (PB) has the barrier with $h_{2}=-8 \mathrm{MeV}$ (see table 1 ). Figure 5 shows how the energy spectra of the two partner bands, the ground band (g) and the side band (s), calculated with the cores PW 


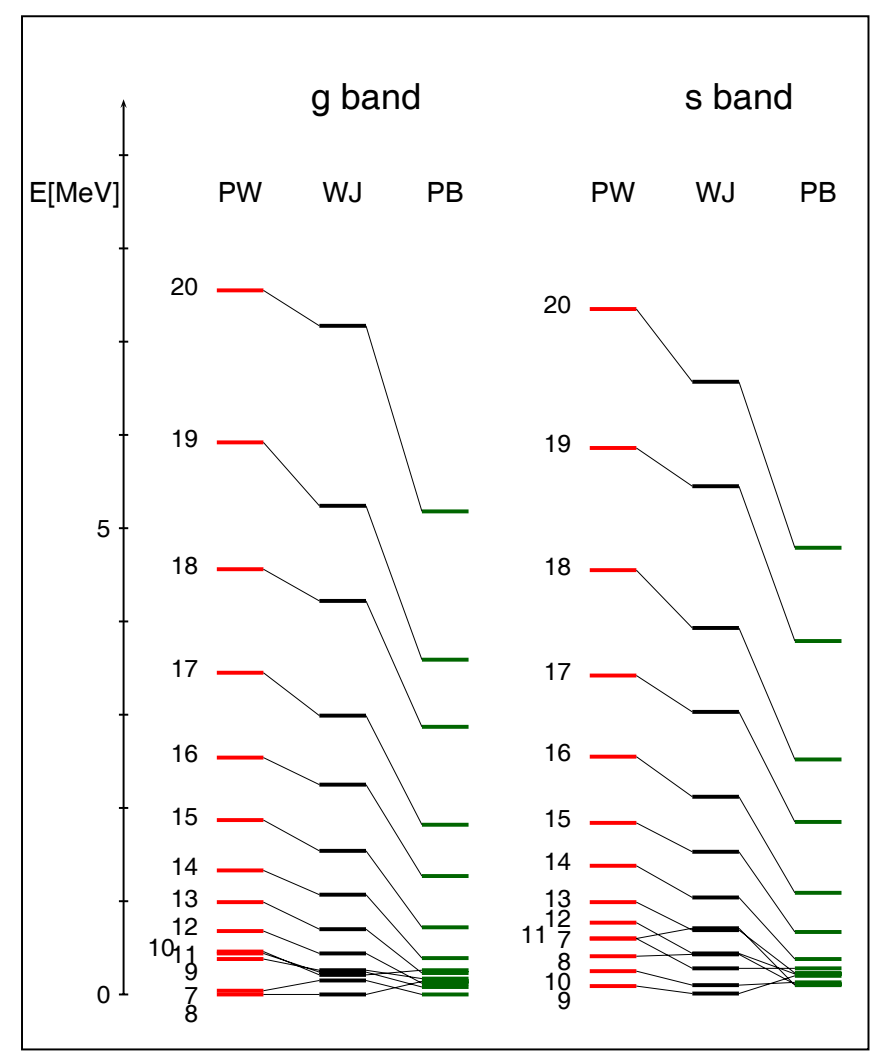

Fig. 5. (Color online) Ground (g) and side (s) partner bands calculated for different cores: the core with the potential energy well (PW), the soft core (WJ) and the core with the potential energy barrier $(\mathrm{PB})$.

and $\mathrm{PB}$ change in comparison with the bands calculated with the WJ core $\left(h_{2}=0\right)$. The convention of grouping the calculated energy levels into bands $g$ or s, which we use here, is explained in detail in ref. [22]. It is seen that both bands are more and more stretched when the $\gamma$-softness of the core decreases (the softness of the barrier can be thought negative). The energies $E\left(I_{\mathrm{b}}\right)$ of the levels within the ground band $(b=g)$ and the side band $(b=s)$ as functions of spin $I_{\mathrm{b}}$ are compared to each other in fig. 6 . The average energy splitting

$$
\Delta E_{\mathrm{av}}=\frac{1}{n} \sum_{I}\left|E\left(I_{\mathrm{s}}\right)-E\left(I_{\mathrm{g}}\right)\right|,
$$

where $n$ is the number of considered spins in the band $(n=13$, or 14$)$, is small and equal to $\Delta E_{\mathrm{av}}=0.13 \mathrm{MeV}$, $0.17 \mathrm{MeV}, 0.12 \mathrm{MeV}$ for cores $\mathrm{PB}$, WJ, PW, respectively. The so-called energy staggering parameter $S\left(I_{\mathrm{b}}\right)=$ $\left[E\left(I_{\mathrm{b}}\right)-E\left(I_{\mathrm{b}}-1\right)\right] /\left(2 I_{\mathrm{b}}\right)$ for the three cases of the core are shown in fig. 7. The spin dependence of $S\left(I_{\mathrm{b}}\right)$ is very similar for both bands $b$ which means in particular that the moments of inertia of these bands are very similar.

The calculated magnetic dipole moments $\mu\left(I_{\mathrm{b}}\right)$ of the odd-odd nucleus do not depend on the details of the core. This is illustrated in fig. 8 where the values of $\mu\left(I_{\mathrm{g}}\right)$ in the states of the ground band of the odd-odd nucleus with different cores are shown. Similar values of $\mu\left(I_{\mathrm{s}}\right) \approx \mu\left(I_{\mathrm{g}}\right)$ are obtained for the side band states $I_{\mathrm{s}}$.

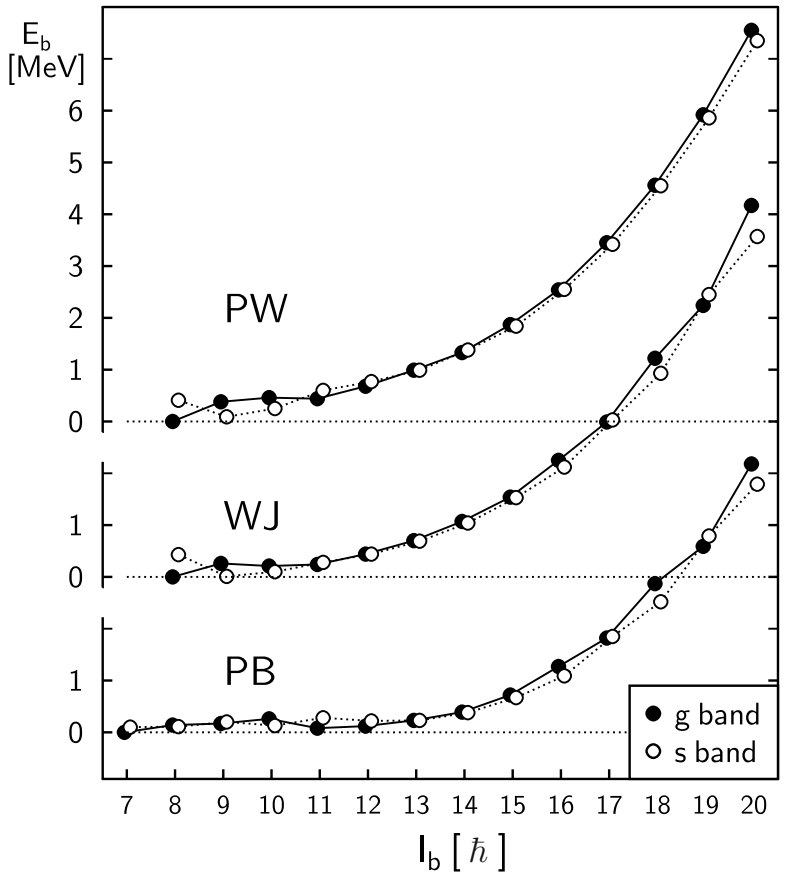

Fig. 6. Energy levels in the ground (g) and side (s) partner bands as functions of spin calculated for different cores: the core with the potential energy well (PW), the soft core (WJ) and the core with the potential energy barrier $(\mathrm{PB})$.

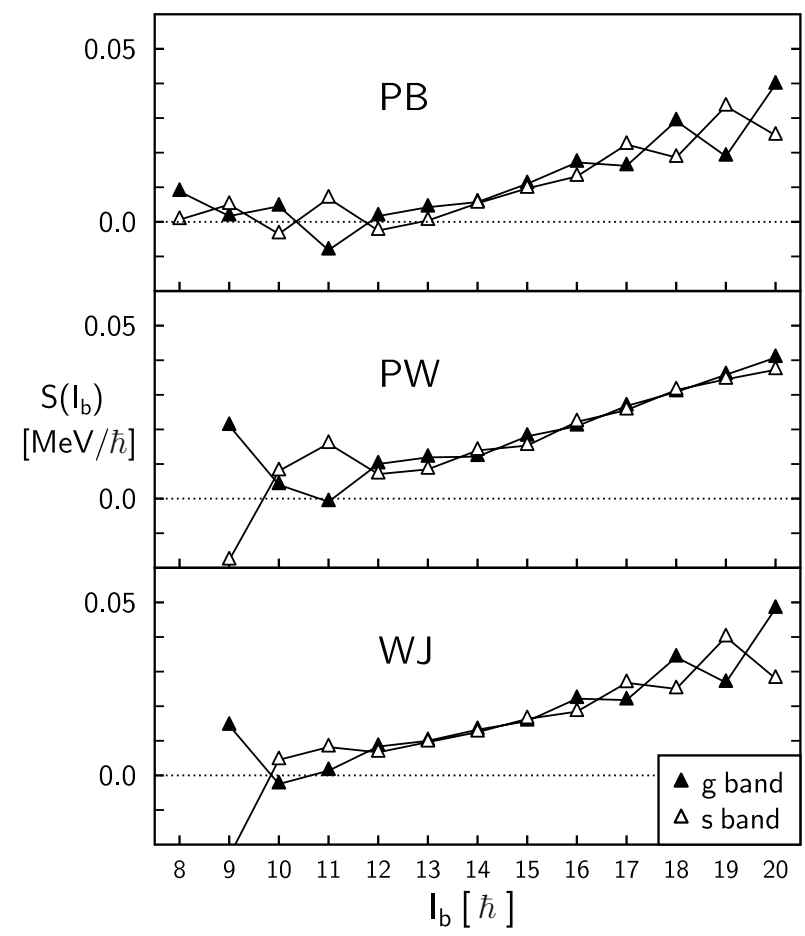

Fig. 7. Energy staggering parameter $S\left(I_{\mathrm{b}}\right)$ of the ground $(\mathrm{b}=$ g) and side $(b=s)$ partner bands for different cores: the core with the potential energy well (PW), the soft core (WJ) and the core with the potential energy barrier $(\mathrm{PB})$.

The electric quadrupole moments of the odd-odd nuclei with the $\alpha$-symmetric cores and the symmetric particle-hole configurations take, as a rule, small values, 


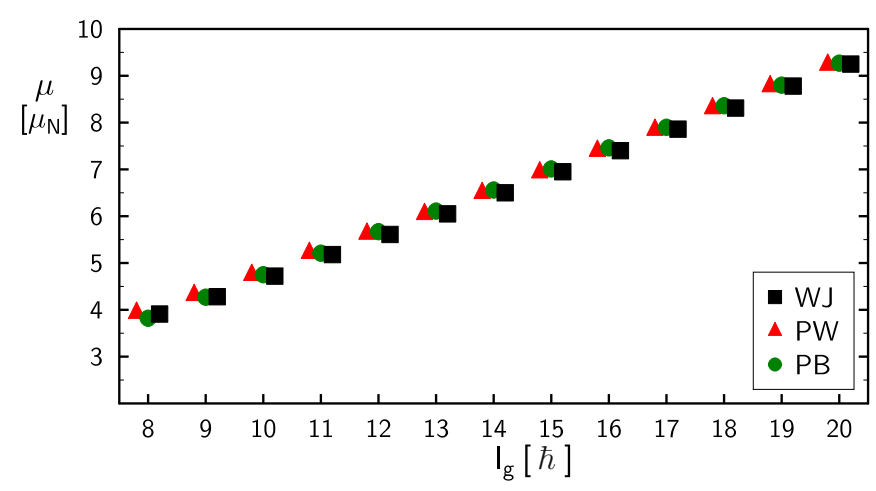

Fig. 8. (Color online) Magnetic dipole moments $\mu\left(I_{\mathrm{g}}\right)$ in the g-band states $I_{\mathrm{g}}$ of the odd-odd nucleus versus spin $I$ calculated for different cores: the core with the potential energy well $(\mathrm{PW})$, the soft core (WJ) and the core with the potential energy barrier $(\mathrm{PB})$.

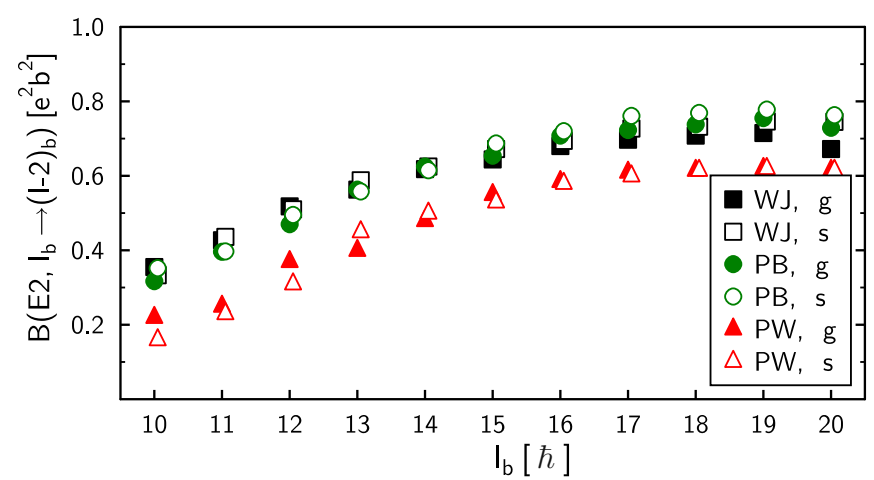

Fig. 9. (Color online) Reduced transition probabilities $B\left(E 2 ; I_{\mathrm{b}} \rightarrow(I-2)_{\mathrm{b}}\right)$ of the stretched intra-band $E 2$ transitions calculated for the $\mathrm{b}=\mathrm{g}$ ground band (full symbols) and the $\mathrm{b}=\mathrm{s}$ side band (open symbols) for the odd-odd nucleus with different cores: the soft core (WJ), the core with the potential energy barrier $(\mathrm{PB})$ and the core with the potential energy well (PW).

less than the single-particle estimate by the order of magnitude (cf. [22]), irrespective of details of the collective potentials of the cores. For instance, the electric quadrupole moment of the $8 \mathrm{~g}$ state is equal to $Q\left(8_{\mathrm{g}}\right)=-0.014$, $-0.031,0.005 \mathrm{eb}$ for the cases WJ, PW, PB, respectively. The corresponding values of the moment in the $20 \mathrm{~g}$ state are equal to $Q\left(20_{\mathrm{g}}\right)=-0.068,-0.055,-0.093 \mathrm{eb}$, respectively.

The reduced transition probabilities $B\left(E 2 ; I_{\mathrm{b}} \rightarrow(I-\right.$ $\left.2)_{\mathrm{b}}\right)$ of the stretched $(\Delta I=2)$ intra-band $E 2$ transitions within both partner bands $(\mathrm{b}=\mathrm{g}, \mathrm{s})$ calculated for the odd-odd nucleus with considered cores are shown in fig. 9 . It is seen that their values for both bands and a given initial spin $I$ are close to each other. The transition probabilities for the nucleus with the core PB (with the potential energy barrier) are close to the corresponding probabilities for the $\gamma$-soft core (WJ) whereas those for the core PW (with the potential energy well) are smaller.

The transitions between the states with $\Delta I=1$ are of the mixed $M 1+E 2$ multipolarity. Figure 10 shows the
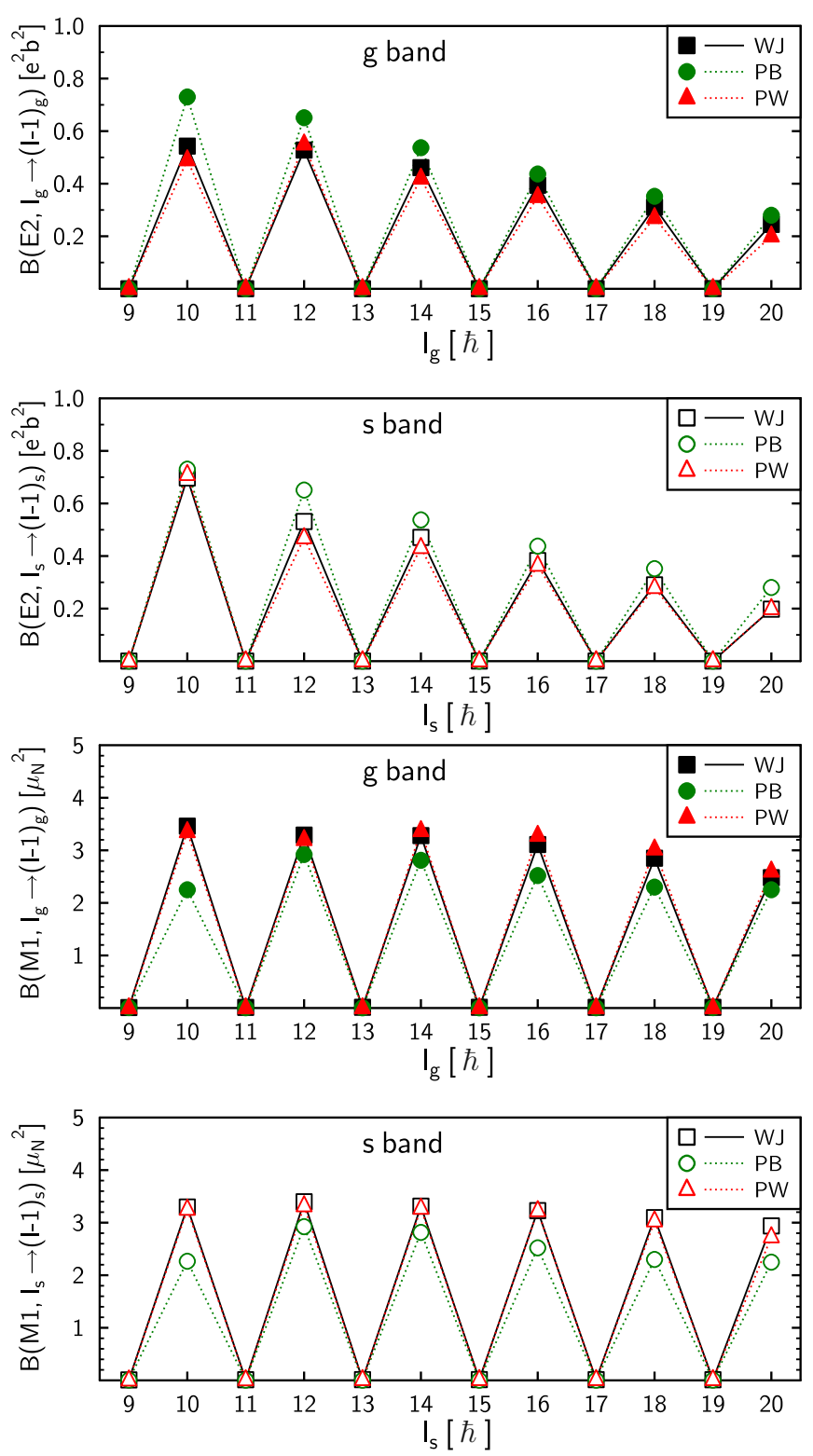

Fig. 10. (Color online) Reduced transition probabilities of the $\Delta I=1$ intra-band electromagnetic transitions in the $\mathrm{g}$ and $\mathrm{s}$ bands calculated with the WJ core (squares), the PB one (circles) and the PW core (triangles). The two upper panels show $B\left(E 2 ; I_{\mathrm{b}} \rightarrow(I-1)_{\mathrm{b}}\right)$ for $\mathrm{b}=\mathrm{g}, \mathrm{s}$. The two lower panels show $B\left(M 1 ; I_{\mathrm{b}} \rightarrow(I-1)_{\mathrm{b}}\right)$ for $\mathrm{b}=\mathrm{g}$, s.

intra-band $\Delta I=1$ transitions calculated for the cases of the PW (with potential well) and the PB (with potential barrier) core. In both cases the $B(E 2)$ and $B(M 1)$ values in the ground $(\mathrm{g})$ and the side $(\mathrm{s})$ band are close to each other, and a strong staggering of these values in both bands appears. We observe a similar phenomenon for the inter-band transitions connecting the side and ground bands. This is shown in fig. 11. Let us notice that the staggering of intra-band and inter-band transitions is in the opposite phase (cf. $[17,22]$ ). In all cases of the $\Delta I=1$ transitions the corresponding values of reduced probabilities are similar to those for the soft WJ core (cf. [22]). We 

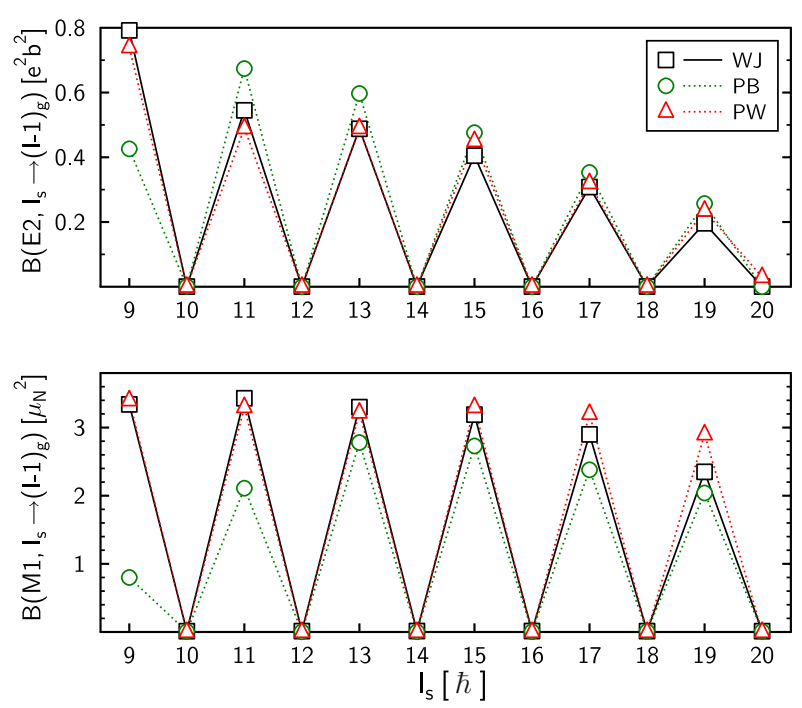

Fig. 11. (Color online) Reduced transition probabilities of the $\Delta I=1$ inter-band electromagnetic transitions from the side to the ground band calculated with the WJ, the PB and the PW core. Upper panel $-B\left(E 2 ; I_{\mathrm{s}} \rightarrow(I-1)_{\mathrm{g}}\right)$; lower panel $-B\left(M 1 ; I_{\mathrm{s}} \rightarrow(I-1)_{\mathrm{g}}\right)$.

conclude that in all the three cases of the core the energy splitting of the partner bands, the differences in values of the electromagnetic moments and transition rates between both bands are small and the $B(M 1)$ and $B(E 2)$ of the intra- and inter-band transitions exhibit a strong and regular staggering. It suggests that the $S$-symmetric odd-odd nuclei, regardless of the detailed properties of the core, manifest the features similar to those characteristic for the chirality phenomenon.

\subsubsection{Proton and neutron on different orbitals}

Let us see how do the results of sect. 3.2.1 change when the proton and the neutron-hole occupy different singleparticle orbitals. In the present calculation we consider the two examples of the particle-hole configuration when the proton is still on the $\pi 1 h_{11 / 2}$ orbital while the neutronhole can occupy either the $\nu 1 g_{9 / 2}^{-1}$ or the $\nu 1 f_{7 / 2}^{-1}$ orbital. In such cases the proton-neutron symmetry - and, in consequence, the combined symmetry $S-$ is broken. The calculations are performed with the WJ soft core as we know that the rigidity of the core does not influence the conclusions regarding the chirality.

Figure 12 shows the partner bands for the three configurations of the valence particles: $\pi 1 h_{11 / 2} \otimes \nu 1 h_{11 / 2}^{-1}(\pi h \otimes$ $\nu h), \pi 1 h_{11 / 2} \otimes \nu 1 g_{9 / 2}^{-1}(\pi h \otimes \nu g)$ and $\pi 1 h_{11 / 2} \otimes \nu 1 f_{7 / 2}^{-1}(\pi h \otimes$ $\nu f)$. We see that the energies of the levels change distinctly according to how the particle-hole configuration is. The average splitting of eq. (28) is equal to $\Delta E_{\mathrm{av}}=0.23 \mathrm{MeV}$ and $0.35 \mathrm{MeV}$ for configuration $h \otimes g$ and $h \otimes f$, respectively. In general, it can be said that the average splitting of the ground and the side band is bigger in the cases of broken $C_{\pi \nu}$ symmetry than in the case of sect. 3.2.1

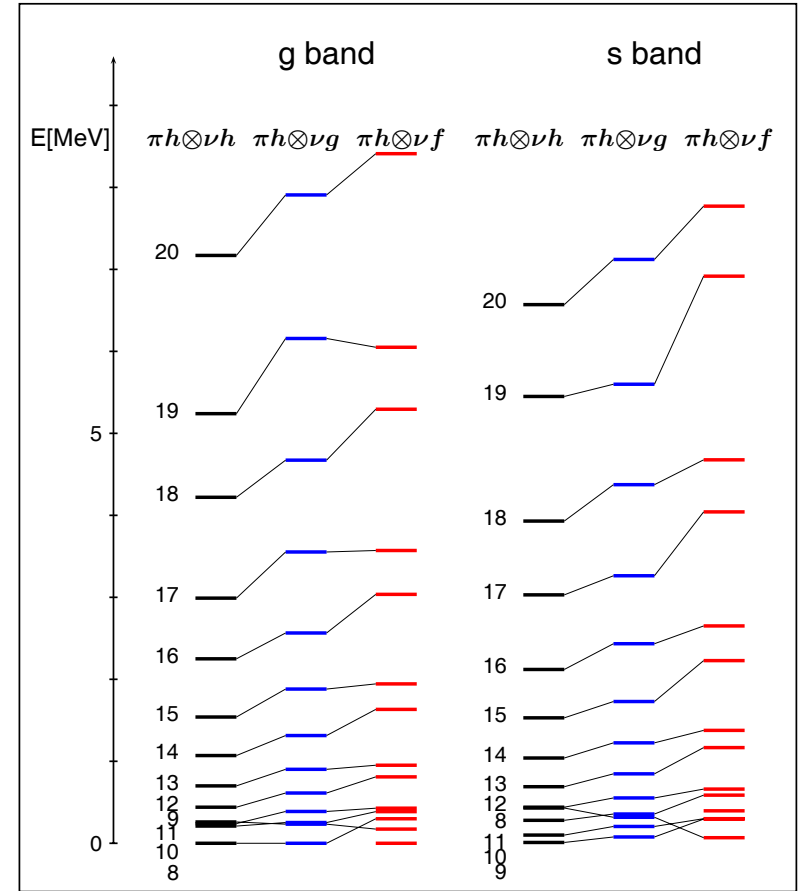

Fig. 12. (Color online) Ground (g) and side (s) bands calculated for the following particle-hole configurations: $\pi 1 h_{11 / 2} \otimes$ $\nu 1 h_{11 / 2}^{-1}(\pi h \otimes \nu h), \pi 1 h_{11 / 2} \otimes \nu 1 g_{9 / 2}^{-1}(\pi h \otimes \nu g)$ and $\pi 1 h_{11 / 2} \otimes$ $\nu 1 f_{7 / 2}^{-1}(\pi h \otimes \nu f)$.

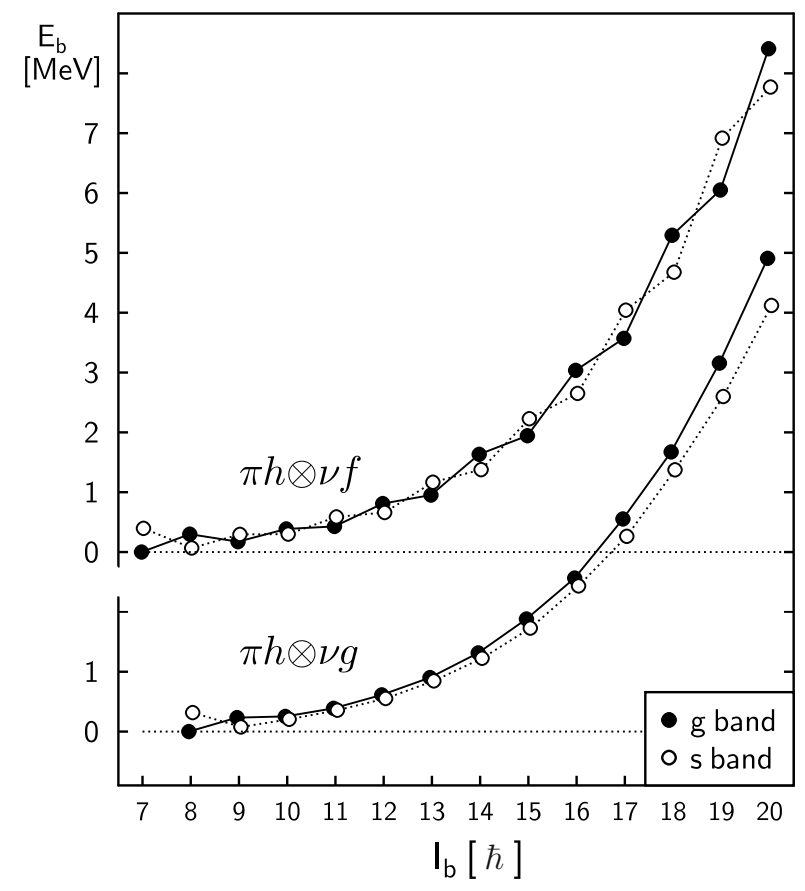

Fig. 13. Energy levels of the ground (g) and side (s) bands as functions of the spin calculated for the following particle-hole configurations: $\pi 1 h_{11 / 2} \otimes \nu 1 g_{9 / 2}^{-1}(\pi h \otimes \nu g)$ and $\pi 1 h_{11 / 2} \otimes \nu 1 f_{7 / 2}^{-1}$ $(\pi h \otimes \nu f)$. 


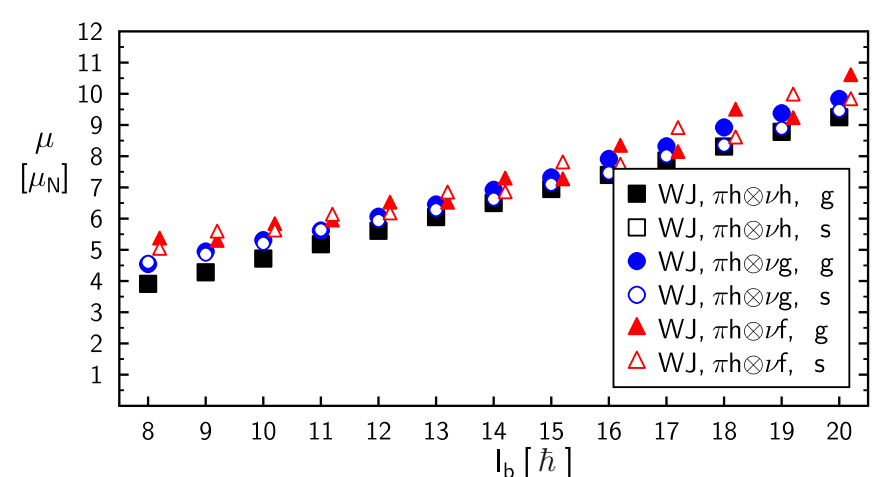

Fig. 14. (Color online) Magnetic dipole moments $\mu\left(I_{\mathrm{b}}\right)$ in the states of the ground and side bands for the particlehole configurations: $\pi 1 h_{11 / 2} \otimes \nu 1 h_{11 / 2}^{-1}, \pi 1 h_{11 / 2} \otimes \nu 1 g_{9 / 2}^{-1}$ and $\pi 1 h_{11 / 2} \otimes \nu 1 f_{7 / 2}^{-1}$.

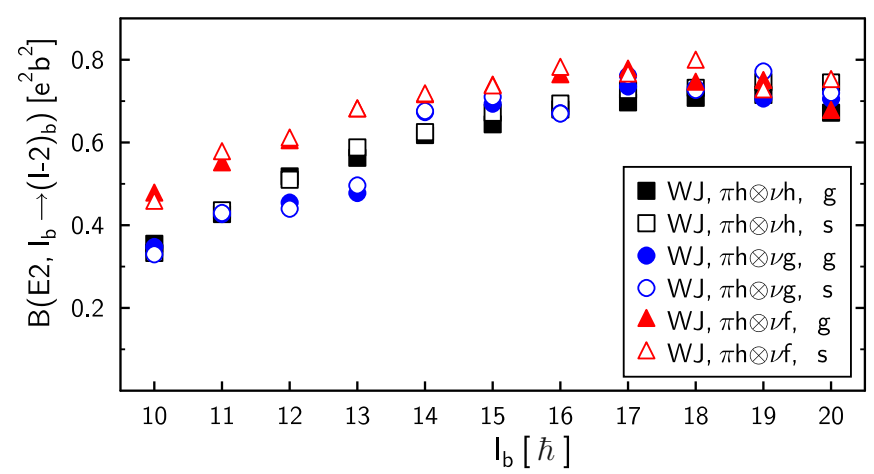

Fig. 15. (Color online) Reduced transition probabilities $B\left(E 2 ; I_{\mathrm{b}} \rightarrow(I-2)_{\mathrm{b}}\right)$ of the stretched intra-band $E 2$ transitions within the $\mathrm{b}=\mathrm{g}$ ground band and the $\mathrm{b}=\mathrm{s}$ side band for the particle-hole configurations: $\pi 1 h_{11 / 2} \otimes \nu 1 h_{11 / 2}^{-1}$, $\pi 1 h_{11 / 2} \otimes \nu 1 g_{9 / 2}^{-1}$ and $\pi 1 h_{11 / 2} \otimes \nu 1 f_{7 / 2}^{-1}$.
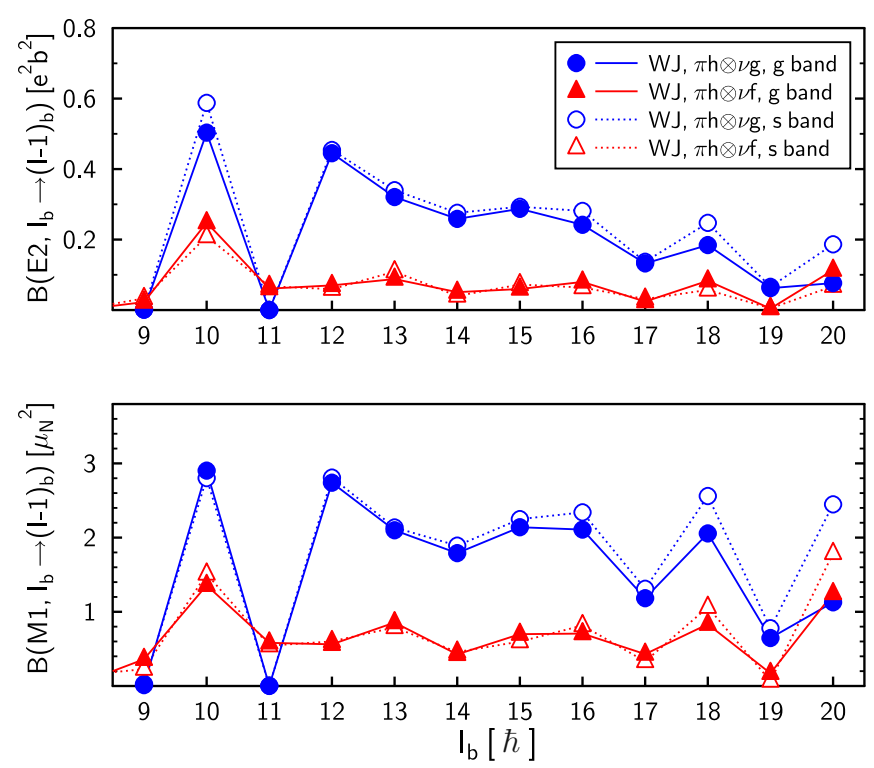

Fig. 16. (Color online) Reduced transition probabilities of the $\Delta I=1$ intra-band electromagnetic transitions within the $\mathrm{g}$ (full symbols) and s (open symbols) bands for the particle-hole configurations: $\pi 1 h_{11 / 2} \otimes \nu 1 g_{9 / 2}^{-1}$ and $\pi 1 h_{11 / 2} \otimes \nu 1 f_{7 / 2}^{-1}$.
Table 2. Contributions of $s= \pm 1$ to the wave functions of the odd-odd nucleus and the electric quadrupole moments $Q$ in the ground $(\mathrm{b}=\mathrm{g})$ and the side $(\mathrm{b}=\mathrm{s})$ band states, $I_{\mathrm{g}}$ and $I_{\mathrm{s}}$, respectively, with $I=8$ and $I=20$ calculated for the following particle-hole configurations: $\pi 1 h_{11 / 2} \otimes \nu 1 h_{11 / 2}^{-1}$ $(\pi h \otimes \nu h), \pi 1 h_{11 / 2} \otimes \nu 1 g_{9 / 2}^{-1}(\pi h \otimes \nu g)$ and $\pi 1 h_{11 / 2} \otimes \nu 1 f_{7 / 2}^{-1}$ $(\pi h \otimes \nu f)$.

\begin{tabular}{crrrr}
\hline$I_{\mathrm{b}}$ & & $\pi h \otimes \nu h$ & $\pi h \otimes \nu g$ & $\pi h \otimes \nu f$ \\
\hline $8_{\mathrm{g}}$ & $s=+1$ & 1.00 & 0.82 & 0.60 \\
& $s=-1$ & 0.00 & 0.18 & 0.40 \\
& $Q[e \mathrm{~b}]$ & -0.014 & -0.480 & -0.852 \\
\hline $8_{\mathrm{s}}$ & $s=+1$ & 0.00 & 0.28 & 0.49 \\
& $s=-1$ & 1.00 & 0.72 & 0.51 \\
& $Q[e \mathrm{~b}]$ & 0.019 & -0.455 & -0.721 \\
\hline $20_{\mathrm{g}}$ & $s=+1$ & 1.00 & 0.96 & 0.30 \\
& $s=-1$ & 0.00 & 0.04 & 0.70 \\
& $Q[e \mathrm{~b}]$ & -0.068 & -0.082 & -0.157 \\
\hline $20_{\mathrm{s}}$ & $s=+1$ & 0.00 & 0.04 & 0.71 \\
& $s=-1$ & 1.00 & 0.96 & 0.30 \\
& $Q[e \mathrm{~b}]$ & -0.063 & -0.256 & -0.506 \\
\hline
\end{tabular}
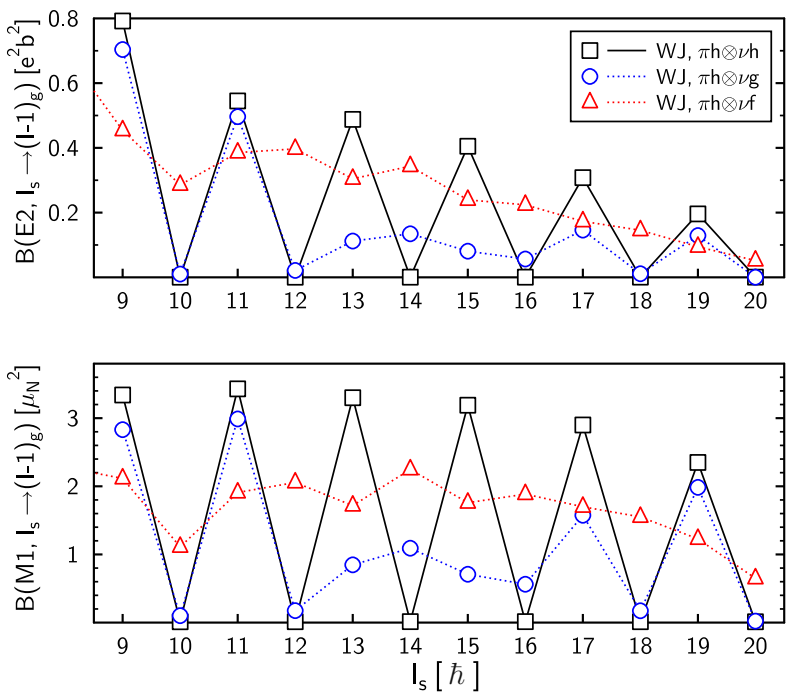

Fig. 17. (Color online) Reduced transition probabilities of the $\Delta I=1$ inter-band electromagnetic transitions from the $\mathrm{s}$ to the $\mathrm{g}$ band for the particle-hole configurations: $\pi 1 h_{11 / 2} \otimes \nu 1 g_{9 / 2}^{-1}$ and $\pi 1 h_{11 / 2} \otimes \nu 1 f_{7 / 2}^{-1}$.

where $C_{\pi \nu}$ is conserved. The calculated energies of the gand s-band levels are compared with one another in fig. 13 for the two considered particle-hole configurations.

In general, the magnetic dipole moments and the stretched E2 intra-band transitions depend obviously on the configuration of the valence particles but differences in their values between the ground and side bands in the cases of broken $C_{\pi \nu}$ are only a bit bigger than those for the conserved $C_{\pi \nu}$-symmetry. The magnetic dipole moments of the ground- and the side-band states for the particle- 
Table 3. Parameters $V_{C}, d, b_{0}$ of the Bohr Hamiltonian selected for each assumed pair of parameters $h_{1}$ and $b_{1}$. The groundstate mean values $\langle\beta\rangle$ and $\langle\gamma\rangle$ of the deformations $\beta$ and $\gamma$, respectively, and energy $E\left(2_{1}^{+}\right)$of the first-excited state and the reduced transition probability $B\left(E 2 ; 2_{1}^{+} \rightarrow 0_{1}^{+}\right)$calculated with these parameters are also shown.

\begin{tabular}{ccccccccc}
\hline $\begin{array}{c}h_{1} \\
(\mathrm{MeV})\end{array}$ & $\begin{array}{c}b_{1} \\
\left(\mathrm{MeV}^{-1}\right)\end{array}$ & $\begin{array}{c}V_{C} \\
(\mathrm{MeV})\end{array}$ & $d$ & $\begin{array}{c}b_{0} \\
\left(\mathrm{MeV}^{-1}\right)\end{array}$ & & $\langle\beta\rangle$ & $\langle\gamma\rangle$ & \multicolumn{2}{c}{$E\left(2_{1}^{+}\right)$} & $\begin{array}{c}B\left(E 2 ; 2_{1}^{+} \rightarrow 0_{1}^{+}\right) \\
(\text {degrees })\end{array}$ & $(\mathrm{MeV})$ & $\left(e^{2} b^{2}\right)$ \\
\hline 0 & 0 & 109.5 & 0.251 & 105 & 0.243 & 30.0 & 0.355 & 0.282 \\
1 & 0 & 100 & 0.18 & 90.5 & 0.244 & 23.9 & 0.356 & 0.270 \\
0 & 60 & 129 & 0.251 & 105 & 0.221 & 23.4 & 0.353 & 0.223 \\
\hline
\end{tabular}

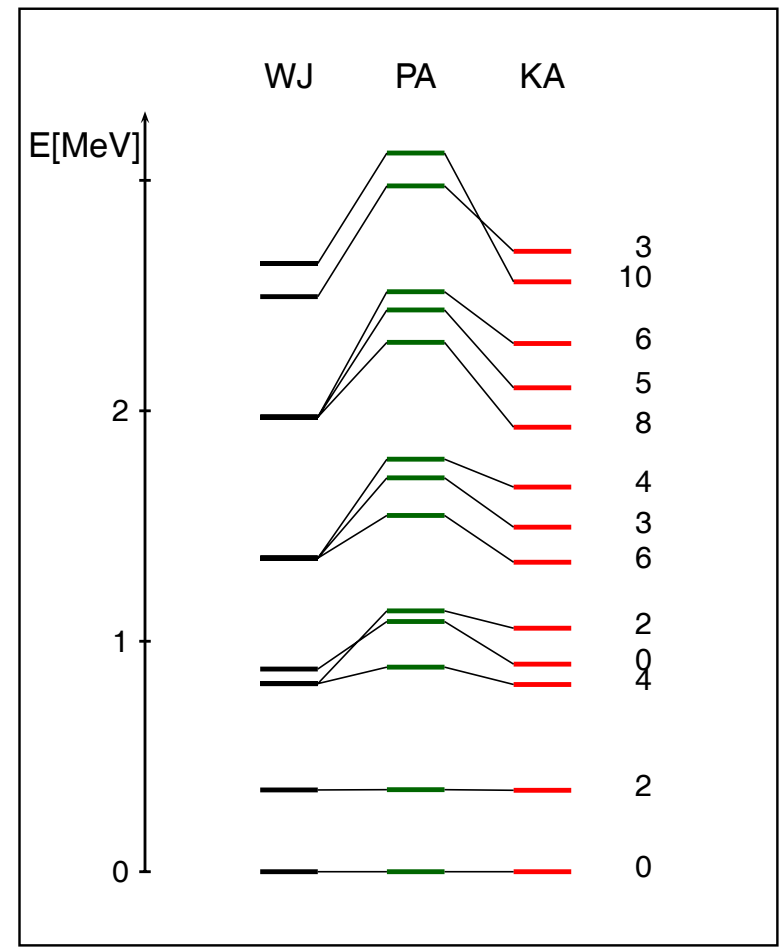

Fig. 18. (Color online) Splitting of the WJ seniority multiplets calculated for $h_{1}=1 \mathrm{MeV}, b_{1}=0(\mathrm{PA})$ and $h_{1}=0, b_{1}=$ $60 / \mathrm{MeV}(\mathrm{KA})$.

hole configurations in question are shown in fig. 14. Their values depend weakly on the configuration which is understandable because the single-particle magnetic moment of the neutron-hole in the $g_{9 / 2}$ and $f_{7 / 2}$ orbitals is the same as in both cases $j=l+1 / 2$. The stretched intraband $E 2$ transitions in both bands for the considered $C_{\pi \nu^{-}}$ asymmetric configurations are compared to the symmetric configuration in fig. 15.

Since the $P_{\alpha}$-symmetry is still conserved, it may seem that the electric quadrupole moments remain small and, as before, depend weakly on the particle-hole configuration. However, breaking the $C_{\pi \nu}$-symmetry leads to breaking of the $S$-symmetry and the components with the two different values of $s$ enter the wave functions of the oddodd nucleus. As a consequence of the $s$ mixing, the diagonal matrix element of $\mathcal{M}(E 2)$ in the state of the oddodd nucleus contains matrix elements of $M(E 2)$ between

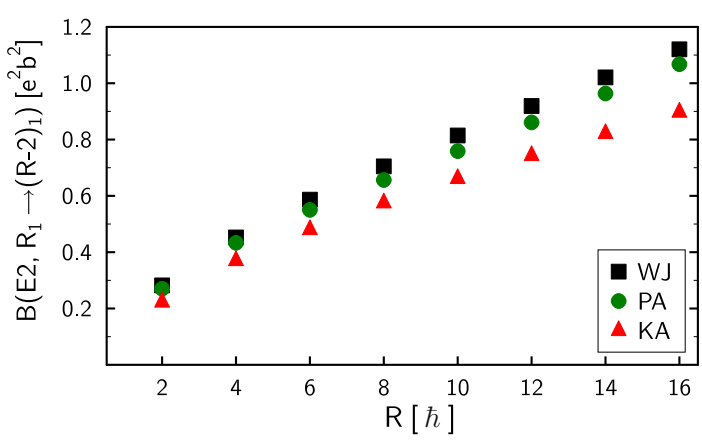

Fig. 19. (Color online) Reduced transition probabilities $B\left(E 2 ; R_{1}^{+} \rightarrow(R-2)_{1}^{+}\right)$calculated for $h_{1}=1 \mathrm{MeV}, b_{1}=0$ $(\mathrm{PA})$ and $h_{1}=0, b_{1}=60 / \mathrm{MeV}(\mathrm{KA})$.

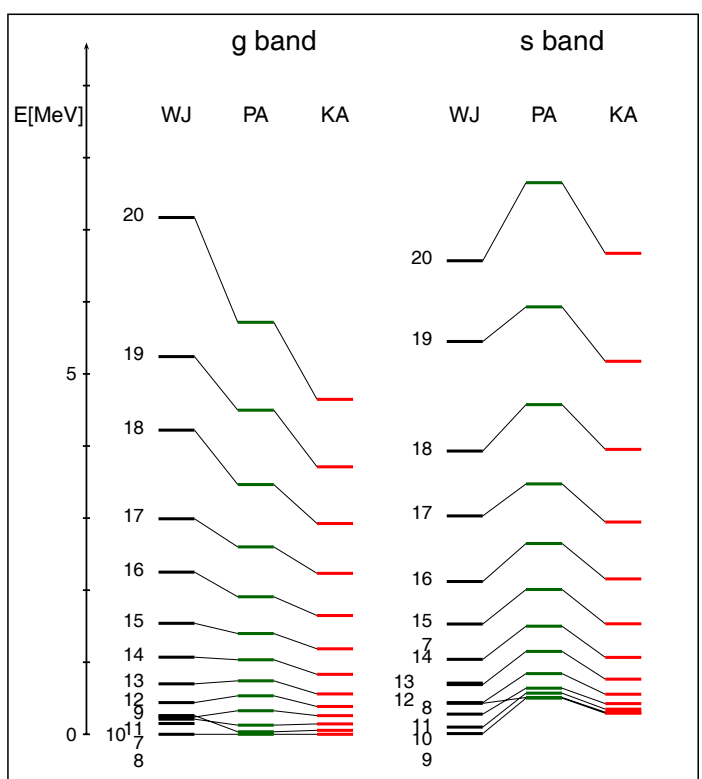

Fig. 20. (Color online) Partner bands in the odd-odd nucleus with the soft WJ core and the $\alpha$-asymmetric PA and KA cores.

the core states with the different $\alpha$-parities $p$, which are relatively large. Therefore, the absolute values of the electric quadrupole moments and their differences between the ground band and the side band states at the broken $C_{\pi \nu^{-}}$ symmetry are bigger than those at the conserved symmetry. It can be read from table 2 where the $s$ mixing and the values of the electric quadrupole moments for selected 


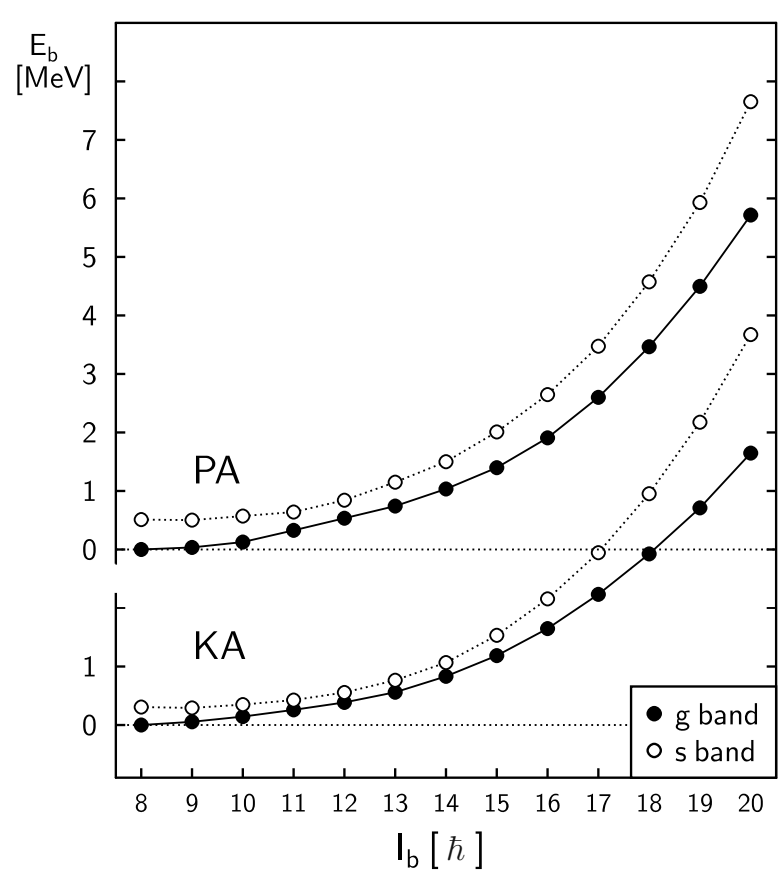

Fig. 21. Energy levels of the $g$ and $s$ bands as functions of spin in the odd-odd nucleus with the $\alpha$-asymmetric PA and KA cores.

Table 4. Electric quadrupole moments $Q\left(I_{\mathrm{b}}\right)$ of the odd-odd nucleus in the ground- and the side-band states with $I=8$ and $I=20$ calculated for the three cores: the soft WJ core, the core PA with the potential $\alpha$-asymmetry and the core KA with the $\alpha$-asymmetry in the kinetic energy.

\begin{tabular}{crrrr}
\hline Core & $Q\left(8_{\mathrm{g}}\right)$ & $Q\left(20_{\mathrm{g}}\right)$ & $Q\left(8_{\mathrm{s}}\right)$ & $Q\left(20_{\mathrm{s}}\right)$ \\
& $(e \mathrm{~b})$ & $(e \mathrm{~b})$ & $(e \mathrm{~b})$ & $(e \mathrm{~b})$ \\
\hline WJ & -0.014 & -0.068 & 0.019 & -0.063 \\
PA & 0.341 & -1.39 & 0.637 & -0.99 \\
KA & 0.336 & -1.64 & 0.521 & -1.10 \\
\hline
\end{tabular}

states in both bands are listed for the different particlehole configurations.

The $\Delta I=1 M 1$ and $E 2$ intra-band and inter-band transitions within the partner bands for the different particle-hole configurations are shown in figs. 16 and 17 . Breaking of the $C_{\pi \nu}$-symmetry causes that the staggering in values of the transition probabilities becomes weaker and irregular. The values of transition probabilities depend strongly on the particle-hole configuration. In the two considered examples, $\pi h \otimes \nu g$ and $\pi h \otimes \nu f$, the intraband transitions are stronger than the inter-band ones in the former case, and it is the other way round in the latter.

In general, we see that breaking of $C_{\pi \nu}$, apart from the disturbances in the staggering effect, causes a bigger average energy splitting between the partner bands than this for the conserved $C_{\pi \nu}$-symmetry and essential differences in values of the electric quadrupole moments. The reduced

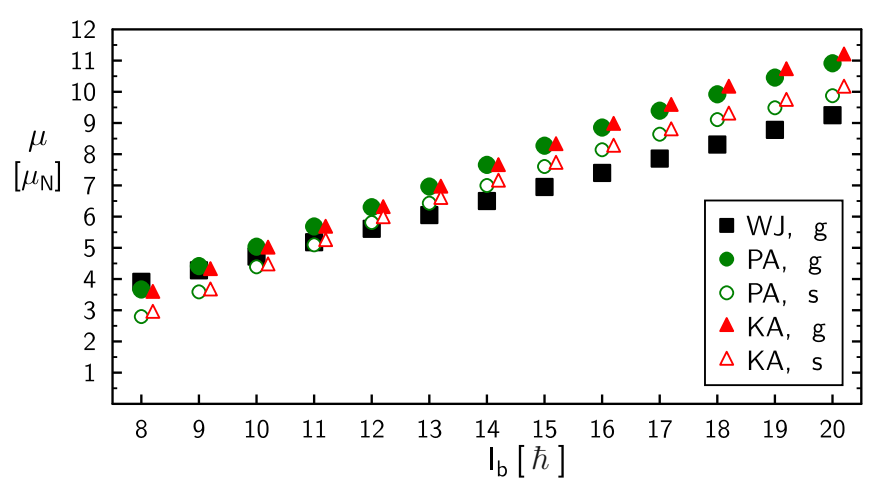

Fig. 22. (Color online) Magnetic dipole moments $\mu\left(I_{\mathrm{b}}\right)$ in the states of the ground and side bands calculated with cores WJ, $\mathrm{PA}$ and KA.

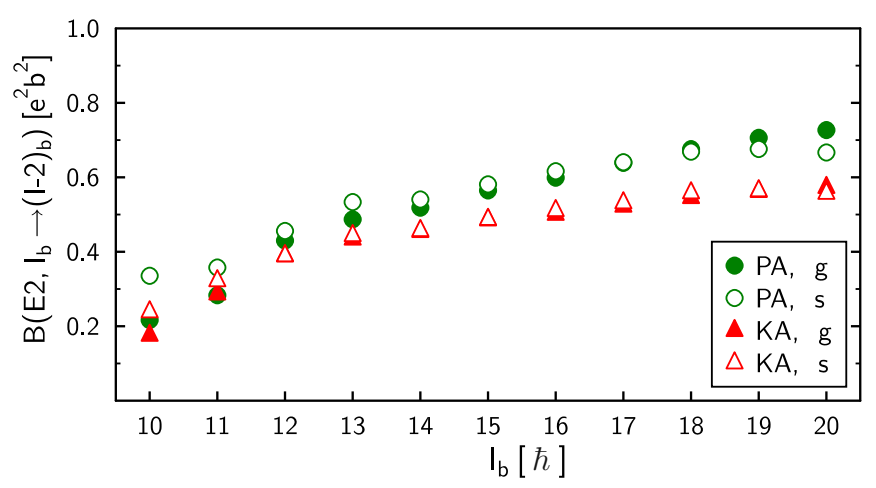

Fig. 23. (Color online) Reduced transition probabilities of the $\Delta I=2$ intra-band $E 2$ transitions in the $\mathrm{g}$ and $\mathrm{s}$ bands calculated with the PA core (circles) and the KA core (triangles).

intra- and inter-band $E 2$ and $M 1$ transition probabilities strongly differ from each other.

\subsection{The $\alpha$-asymmetric cores}

In the present version of the model Hamiltonian (4) of the core the $\alpha$-symmetry is broken for $h_{1} \neq 0$ (the $\alpha$ asymmetric potential) and/or $b_{1} \neq 0$ (the $\alpha$-asymmetric kinetic energy). Below we present two such examples of the cores with broken $\alpha$-symmetry. We take the Bohr Hamiltonian (4) with the $B_{\beta \beta}=250 / \mathrm{MeV}, h_{2}=0$, $G=8.605 \mathrm{MeV}$ and the two versions of parameters $h_{1}$ and $b_{1}$, namely: i) $h_{1}=1 \mathrm{MeV}, b_{1}=0$ (PA), ii) $h_{1}=0$, $b_{1}=60 / \mathrm{MeV}(\mathrm{KA})$. The three remaining parameters of the Hamiltonian, $b_{0}, V_{C}$ and $d$ have been selected for each pair of parameters $\left(h_{1}, b_{1}\right)$ in order to keep the values of $E\left(2_{1}^{+}\right)$and $B\left(E 2 ; 2_{1}^{+} \rightarrow 0_{1}^{+}\right)$close to the experimental values for ${ }_{56}^{128} \mathrm{Ba}$. The obtained values of $E\left(2_{1}^{+}\right)$and $B\left(E 2 ; 2_{1}^{+} \rightarrow 0_{1}^{+}\right)$together with $\langle\beta\rangle$ and $\langle\gamma\rangle$, the mean values of $\beta$ and $\gamma$, for each set of parameters of the Hamiltonian are given in table 3 .

The mean values of $\gamma$ about $23^{\circ}$ indicate a weak breaking of the $\alpha$-symmetry. The energy spectra of the cores for the two different asymmetries are shown in fig. 18. The WJ seniority multiplets become split when the asymmetry arises. On the other hand, the behaviour of the intra-band 

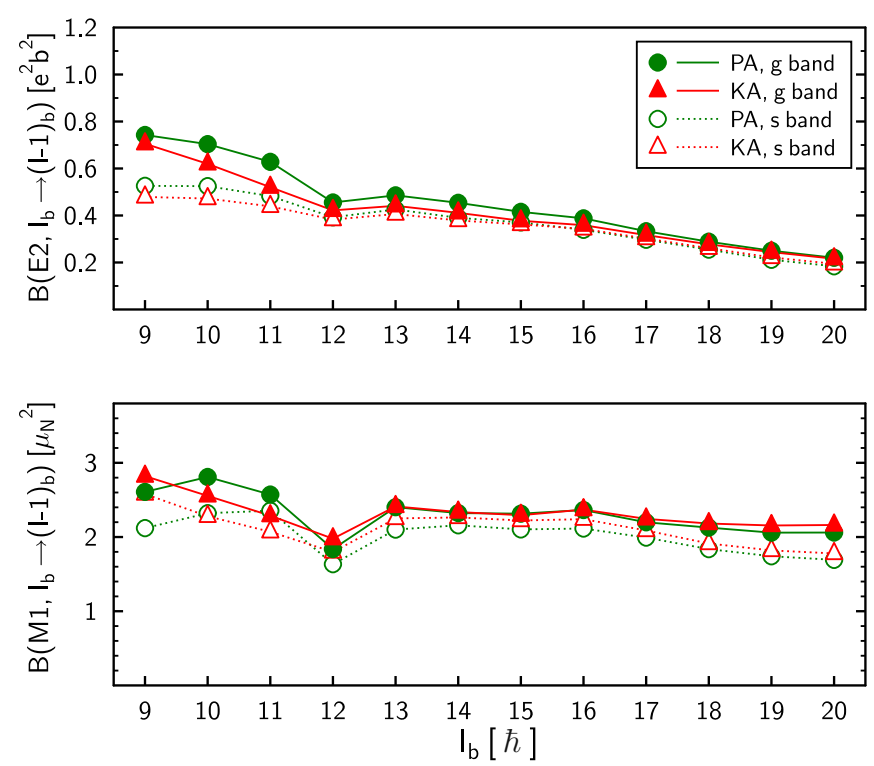

Fig. 24. (Color online) Reduced transition probabilities of the $\Delta I=1$ intra-band electromagnetic transitions in the $\mathrm{b}=\mathrm{g}$ and $\mathrm{b}=\mathrm{s}$ bands calculated with the PA core (circles) and the KA core (triangles). The upper panel shows $B\left(E 2 ; I_{\mathrm{b}} \rightarrow(I-1)_{\mathrm{b}}\right)$ and the lower one $B\left(M 1 ; I_{\mathrm{b}} \rightarrow(I-1)_{\mathrm{b}}\right)$.
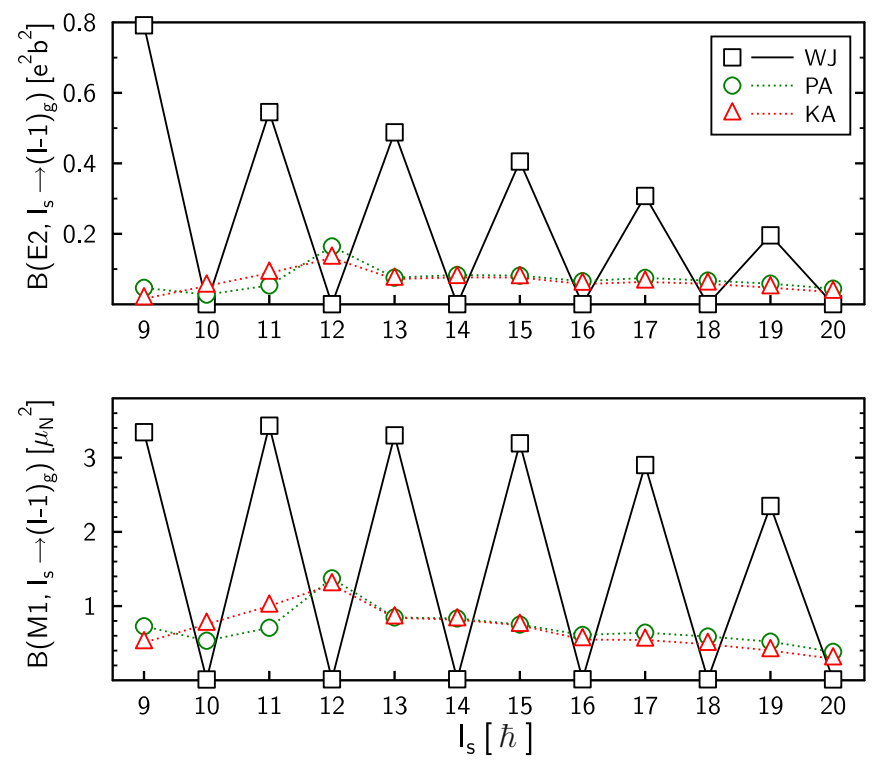

Fig. 25. (Color online) Reduced transition probabilities of the $\Delta I=1$ inter-band electromagnetic transitions from the side to the ground band calculated with the WJ, the PA and the KA core. Upper panel $-B\left(E 2 ; I_{\mathrm{s}} \rightarrow(I-1)_{\mathrm{g}}\right)$; lower panel $-B\left(M 1 ; I_{\mathrm{s}} \rightarrow(I-1)_{\mathrm{g}}\right)$.

stretched $E 2$ transitions within the ground-state band is fairly similar to that in the WJ core. It is seen in fig. 19 where the $B\left(E 2 ; R_{\mathrm{g}} \rightarrow(R-2)_{\mathrm{g}}\right)$ values for the WJ, PA and KA cores are shown.

\subsection{Odd-odd nuclei with the $\alpha$-asymmetric cores}

In order to observe effects of breaking the $\alpha$-symmetry only, it is assumed that the odd proton and neutron- hole in the odd-odd nucleus occupy the same singleparticle orbital $1 h_{11 / 2}$. The particle-hole configuration $\pi 1 h_{11 / 2} \otimes \nu 1 h_{11 / 2}^{-1}$ of the valence particles conserves the proton-neutron symmetry. The partner bands are split much stronger than those of the odd-odd nucleus with the WJ core. It can be easily deduced from fig. 20. The average band splitting $\Delta E_{\mathrm{av}}$ is equal to $0.73 \mathrm{MeV}$ and $0.58 \mathrm{MeV}$ for the cases PA and KA, respectively. The standard band presentation in fig. 21 shows clearly the splitting of the bands. The electric quadrupole moments are obviously bigger than those in the case of soft core. Also, the differences in values of the quadrupole moment between the ground and side bands are quite large. We see these phenomena in table 4 . The differences in the values of the magnetic dipole moments between the $\mathrm{g}$ and $\mathrm{s}$ bands become significant, as is seen in fig. 22 .

The stretched intra-band $E 2$ transitions reflect the trends in such transitions in the cores (fig. 19) and the values of reduced transition probabilities do not differ much in the bands $\mathrm{g}$ and $\mathrm{s}$. It is seen in fig. 23 .

On the other hand, the behaviour of the $\Delta I=1$ intraand inter-band transitions, both $E 2$ and $M 1$ changes dramatically. The characteristic regular and strong staggering of the $B(E 2)$ and $B(M 1)$ values vanishes completely. The all intra-band transitions remain relatively strong while the inter-band ones become all weak. It is seen in figs. 24 and 25 .

In summary, we notice that even a weak $\alpha$-asymmetry of the Bohr Hamiltonian describing the core, both in the potential and in the kinetic energy, causes decay of the staggering of the $M 1$ and E2 intra- and inter-band transition probabilities. Other characteristics like the band splitting, the differences in the electromagnetic moments and the transition probabilities between the bands increase gradually when the $\alpha$-asymmetry increases (cf. [33] where an example of stronger $\alpha$-asymmetry is presented). The main consequence of the $\alpha$-symmetry breaking which causes visible changes in the characteristics of the odd-odd nucleus is the fact that eq. (15) is no longer fulfilled and the diagonal matrix elements of $Q$ do not vanish irrespective of a kind of the $\alpha$-asymmetry of the core Hamiltonian (the asymmetric potential and/or the kinetic energy) and, in particular, the rigidity of the core (see [22]). Therefore, the results of calculations with the $\alpha$-asymmetric cores with different asymmetries will be qualitatively similar.

\section{Conclusions}

Fictitious odd-odd nuclei of $Z=57$ and $N=71(A=128)$ with various cores described by the Bohr collective Hamiltonians and different particle-hole configurations of the odd particles have been investigated in the frame of the Core-Particle-Hole Coupling model. The odd proton and neutron can be treated as the particle and hole, respectively, in the odd-odd nuclei from the $A \sim 130$ region. The sets of parameters of the collective Hamiltonians of the cores have been chosen so as to obtain cores with very different symmetry properties although with the similar 
equilibrium $\beta$-deformation equal to about 0.25 and the energies of the $2_{1}^{+}$state and the $B\left(E 2 ; 2_{1}^{+} \rightarrow 0_{1}^{+}\right)$value close to the experimental values for ${ }^{128} \mathrm{Ba}$. The energy levels with spins $I=8-20$, the $M 1$ and $E 2$ moments and transitions in the odd-odd nuclei have been calculated. The energy levels and the reduced probabilities of transitions between them allow for grouping the states into the two partner bands. Properties of these bands have been examined for the appearance of the characteristics suggested as main signatures of nuclear chiral systems. The conclusion is that a variety of the core-particle-hole systems possessing the combined $S=P_{\alpha} C_{\pi \nu}$ symmetry, irrespective of the rigidity of the core, manifests characteristics usually ascribed to the systems which have broken chiral symmetry. The examples presented here indicate that the breaking of the proton-neutron symmetry $C_{\pi \nu}$ perturbs these characteristics in different ways subject to a particular particle-hole configuration. The average energy splitting of the partner bands increases and the differences between the values of the electromagnetic moments, especially the electric quadrupole moments, for the g- and sbands are enlarged. Staggering of the $\Delta I=1 B(M 1)$ and $B(E 2)$ values is smaller and irregular. The values of the probabilities of the intra- and inter-band transitions differ from each other not only in the phase. Breaking of the $\alpha$ symmetry $P_{\alpha}$ causes a bigger band splitting which grows with the increasing asymmetry. Also, the differences in the electromagnetic moments and in the intra-band $\Delta I=1$ transition probabilities between the bands behave in a similar way. The staggering effect in the $\Delta I=1 M 1$ and $E 2$ transitions disappears and the inter-band transitions become much smaller than the intra-band ones.

The three-body systems investigated here are much more involved and realistic than the classical chiral system with the three mutually perpendicular angular momenta - the triaxial rigid rotor, and particle and hole occupying a high- $j$ orbital. The current work investigates the properties of doublet bands in these systems in the laboratory frame of reference by examining if they manifest various characteristics attributed usually to the systems with broken chiral symmetry. Although the body-fixed frame has been used in the description of the core, it is not easily accessible in the description of the three-body system. It is so because the core characteristics enter the description of the odd-odd nucleus only through the energies of the collective levels and the quadrupole matrix elements within the collective states. In our approach no assumption about the chirality is introduced. The models developed here are capable of describing the same properties of the states of the odd-odd nuclei as they are described in terms of the models which start from the chiral geometry of the angular momentum coupling in the intrinsic frame. We conclude from our investigation that the three-body systems with the $S$-symmetry show the characteristics which are usually treated as the "chirality fingerprints" discussed in the literature. A detailed investigation through the calculation of scalar operators [8] of the angular momentum coupling in such three-body systems is planned as a follow-up of this studies.
This work was performed within the frame of project No. GPOOL/2009/0 and supported in part by the Polish Ministry of Science and Higher Education under Contract No. N N202 328234 as well as the Natural Sciences and Engineering Research Council of Canada under contract No. SAPIN/3716562010 .

Open Access This article is distributed under the terms of the Creative Commons Attribution Noncommercial License which permits any noncommercial use, distribution, and reproduction in any medium, provided the original author(s) and source are credited.

\section{References}

1. S. Frauendorf, J. Meng, Nucl. Phys. A 617, 131 (1997).

2. V.I. Dimitrov, S. Frauendorf, F. Dönau, Phys. Rev. Lett. 84, 5732 (2000).

3. V.I. Dimitrov, F. Dönau, S. Frauendorf, Phys. Rev. C 62, 024315 (2000).

4. P. Olbratowski, J. Dobaczewski, J. Dudek, W. Płóciennik, Phys. Rev. Lett. 93, 052501 (2004).

5. P. Olbratowski, J. Dobaczewski, J. Dudek, Phys. Rev. C 73, 054308 (2006).

6. A. Klein, P. Protopapas, S.G. Rohoziński, K. Starosta, Phys. Rev. C 69, 034338 (2004).

7. S.G. Rohoziński, K. Starosta, Int. J. Mod. Phys. 14, 427 (2005).

8. K. Starosta, C.J. Chiara, D.B. Fossan, T. Koike, T.T.S. Kuo, D.R. LaFosse, S.G. Rohoziński, Ch. Droste, T. Morek, J. Srebrny, Phys. Rev. C 65, 044328 (2002).

9. T. Koike, K. Starosta, C.J. Chiara, D.B. Fossan, D.R. LaFosse, Phys. Rev. C 67, 044319 (2003).

10. J. Peng, J. Meng, S.Q. Zhang, Phys. Rev. C 68, 044324 (2003).

11. S.Q. Zhang, B. Qi, S.Y. Wang, J. Meng, Phys. Rev. C 75, 044307 (2007).

12. S.Y. Wang, S.Q. Zhang, B. Qi, J. Meng, Phys. Rev. C 75, 024309 (2007).

13. S.Y. Wang, S.Q. Zhang, B. Qi, J. Peng, J.M. Yao, J. Meng, Phys. Rev. C 77, 034314 (2008).

14. E.A. Lawrie, P.A. Vymers, J.J. Lawrie, Ch. Vieu, R.A. Bark, R. Lindsay, G.K. Mabala, S.M. Maliage, P.L. Masiteng, S.M. Mullins, S.H.T. Murray, I. Ragnarsson, T.M. Ramashidzha, C. Schück, J.F. Sharpey-Schafer, O. Shirinda, Phys. Rev. C 78, 021305 (2008).

15. T. Koike, K. Starosta, I. Hamamoto, Phys. Rev. Lett. 93, 172502 (2004).

16. I. Hamamoto, Int. J. Mod. Phys. E 20, 373 (2011).

17. E. Grodner, Int. J. Mod. Phys. E 20, 380 (2011).

18. J. Meng, S.Q. Zhang, J. Phys. G: Nucl. Part. Phys. 37, 064025 (2010).

19. J. Meng, Int. J. Mod. Phys. E 20, 341 (2011).

20. E. Grodner, J. Srebrny, A.A. Pasternak, I. Zalewska, T. Morek, Ch. Droste, J. Mierzejewski, M. Kowalczyk, J. Kownacki, M. Kisieliński, S.G. Rohoziński, T. Koike, K. Starosta, A. Kordyasz, P.J. Napiorkowski, M. WolinnskaCichocka, E. Ruchowska, W. Płóciennik, J. Perkowski, Phys. Rev. Lett. 97, 172501 (2006).

21. E. Grodner, I. Sankowska, T. Morek, S.G. Rohoziński, Ch. Droste, J. Srebrny, A.A. Pasternak, M. Kisieliński, M. Kowalczyk, J. Mierzejewski, A. Król, K. Wrzosek, to be published in Phys. Lett. B. 
22. Ch. Droste, S.G. Rohoziński, L. Próchniak, K. Starosta, E. Grodner, Eur. Phys. J. A 42, 79 (2009).

23. N. Yoshinaga, K. Higashiyama, J. Phys. G: Nucl. Part. Phys. 37, 115104 (2010).

24. L. Próchniak, S.G. Rohoziński, J. Phys. G: Nucl. Part. Phys. 36, 123101 (2009).

25. A.S. Davydov, G.F. Filippov, Nucl. Phys. 8, 237 (1958).

26. L. Wilets, M. Jean, Phys. Rev. 102, 788 (1956).

27. A. Bohr, B.R. Mottelson, Nuclear Structure, Vol. I (Benjamin, New York, Amsterdam, 1969).
28. L. Próchniak, S.G. Rohoziński, Ch. Droste, K. Starosta, Acta Phys. Pol. B 42, 465 (2011).

29. D.J. Rowe, T.A. Welsh, M.A. Caprio, Phys. Rev. C 79, 054304 (2009).

30. D.R. Bés, Nucl. Phys. 10, 373 (1959).

31. G. Rakavy, Nucl. Phys. 4, 289 (1957).

32. A. Bohr, B.R. Mottelson, Nuclear Structure, Vol. II (Benjamin, Reading, MA, 1975).

33. S.G. Rohoziński, L. Próchniak, Ch. Droste, K. Starosta, Int. J. Mod. Phys. E 20, 364 (2011). 